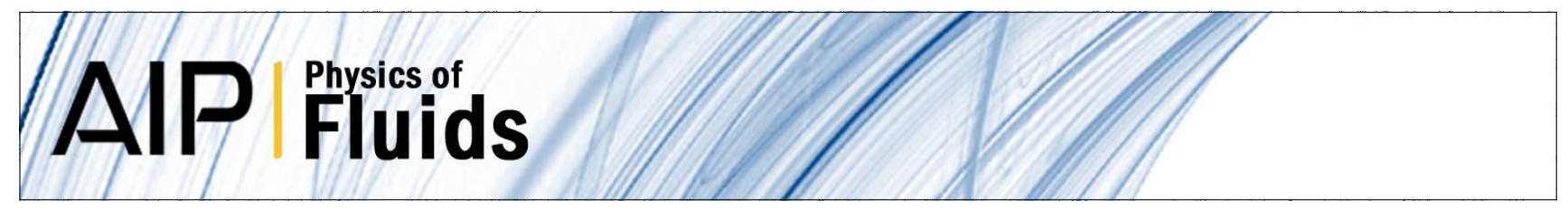

\title{
Effects of continuum breakdown on hypersonic aerothermodynamics for reacting flow
}

Timothy D. Holman and lain D. Boyd

Citation: Phys. Fluids 23, 027101 (2011); doi: 10.1063/1.3541816

View online: http://dx.doi.org/10.1063/1.3541816

View Table of Contents: http://pof.aip.org/resource/1/PHFLE6/v23/i2

Published by the AIP Publishing LLC.

\section{Additional information on Phys. Fluids}

Journal Homepage: http://pof.aip.org/

Journal Information: http://pof.aip.org/about/about_the_journal

Top downloads: http://pof.aip.org/features/most_downloaded

Information for Authors: http://pof.aip.org/authors

\section{ADVERTISEMENT}

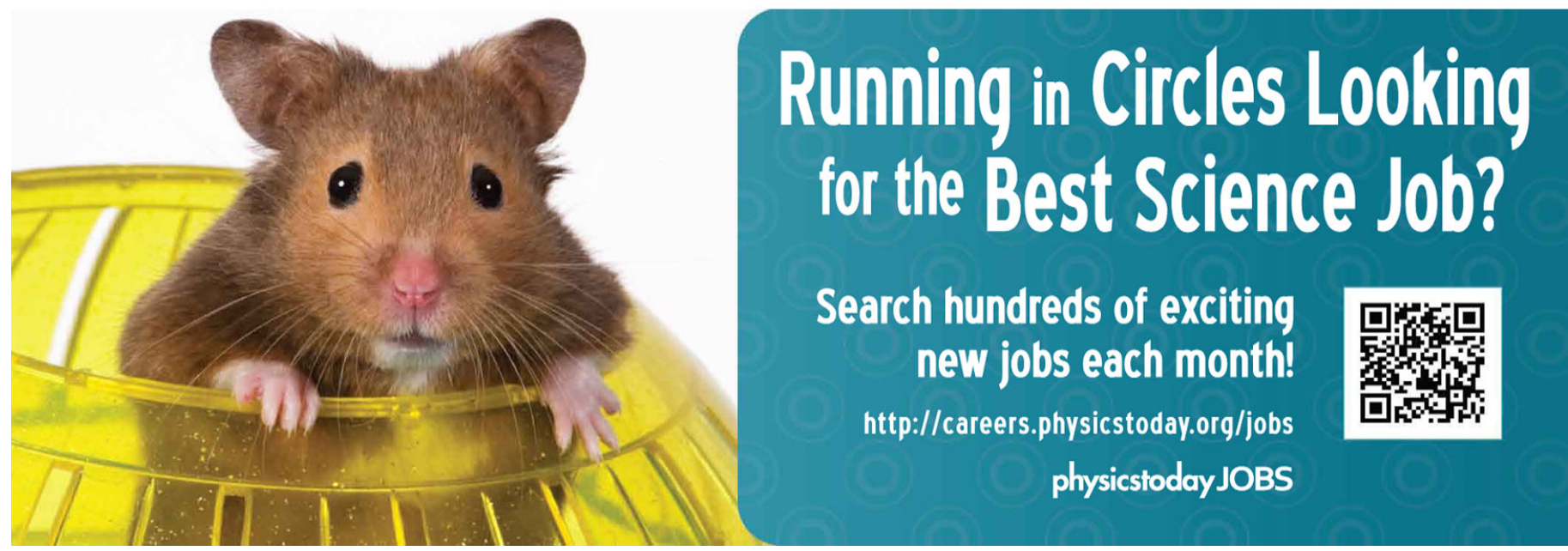




\title{
Effects of continuum breakdown on hypersonic aerothermodynamics for reacting flow
}

\author{
Timothy D. Holman and lain D. Boyd \\ Department of Aerospace Engineering, University of Michigan, Ann Arbor, Michigan 48109, USA
}

(Received 16 August 2010; accepted 16 December 2010; published online 1 February 2011)

\begin{abstract}
This study investigates the effects of continuum breakdown on the surface aerothermodynamic properties (pressure, stress, and heat transfer rate) of a sphere in a Mach 25 flow of reacting air in regimes varying from continuum to a rarefied gas. Results are generated using both continuum [computational fluid dynamics (CFD)] and particle [direct simulation Monte Carlo (DSMC)] approaches. The DSMC method utilizes a chemistry model that calculates the backward rates from an equilibrium constant. A preferential dissociation model is modified in the CFD method to better compare with the vibrationally favored dissociation model that is utilized in the DSMC method. Tests of these models are performed to confirm their validity and to compare the chemistry models in both numerical methods. This study examines the effect of reacting air flow on continuum breakdown and the surface properties of the sphere. As the global Knudsen number increases, the amount of continuum breakdown in the flow and on the surface increases. This increase in continuum breakdown significantly affects the surface properties, causing an increase in the differences between CFD and DSMC. Explanations are provided for the trends observed.
\end{abstract}

(C) 2011 American Institute of Physics. [doi:10.1063/1.3541816]

\section{INTRODUCTION}

A hypersonic vehicle crosses many regimes from rarefied to continuum due to the change in density with altitude during the course of its trajectory through a planet's atmosphere. This variation makes it difficult to simulate the flow since the physical accuracy of computational fluid dynamics (CFD) can breakdown in rarefied flows and the direct simulation Monte Carlo (DSMC) method is computationally expensive in continuum flows. It is difficult and expensive to reproduce these varied flow conditions in ground based experiments and flight tests, so there is a need for computational models that can be utilized for design and development of hypersonic vehicles.

The flow can be characterized by the Knudsen number as given in Eq. (1).

$$
\mathrm{Kn}=\frac{\lambda}{L} \propto \frac{1}{\rho L} .
$$

When the Knudsen number is much less than 1, the flow can be considered to be continuum and therefore should be simulated using traditional computational fluid dynamics techniques by numerically solving the Navier-Stokes equations. However, when the Knudsen number becomes larger, the continuum assumption in the Navier-Stokes equations starts to breakdown. This is due to the fact that these equations are derived from kinetic theory based on the assumption of small perturbations from an equilibrium velocity distribution function; ${ }^{1}$ therefore, CFD only works in near continuum flows. At higher altitudes, where the density is lower giving a larger Knudsen number, only a noncontinuum technique can be used, such as the DSMC method. ${ }^{2}$ In continuum flows over a blunt body, there can be a locally rarefied flow in the shock, the boundary layer, and the wake of the body. As a result, neither CFD nor DSMC can provide a complete computational model across all regimes of a hypersonic vehicle.

In order to identify the areas where the CFD method is in breakdown, the use of a continuum breakdown parameter is needed. Boyd et al. ${ }^{3}$ suggested the use of the maximum gradient length local Knudsen number as a continuum breakdown parameter given in Eq. (2)

$$
\mathrm{Kn}_{G L L}=\frac{\lambda}{Q}\left|\frac{d Q}{d l}\right|,
$$

where the derivative is taken in the direction of maximum gradient and $Q$ is a variable of interest such as density, temperature, or pressure. It has been found that a value of $\mathrm{Kn}_{G L L}$ above 0.05 indicates continuum breakdown has occurred.

The DSMC method can be utilized in any dilute gas flow but becomes prohibitively expensive for low Knudsen number flows. In general, a CFD method is an order of magnitude faster than the DSMC method. Therefore, ways to extend the validity of CFD to higher Knudsen numbers are desirable. It has been found that replacing the no-slip boundary condition typically employed in the CFD method with a velocity slip and temperature jump boundary condition can extend the CFD method into the transition regime. ${ }^{4}$ However, if the flow is too far from continuum, the slip boundary conditions will not help the CFD method and a DSMC method is required for accurate simulation of the flow.

To be able to design a hypersonic vehicle, it is important to be able to predict the surface properties on the vehicle. In order to do this, one must understand how continuum breakdown affects the surface conditions such as heat flux, pressure and shear stress. These surface conditions determine the aerothermodynamic performance of a reentry vehicle. A previous study by Lofthouse et al. ${ }^{5}$ looked at the effect of con- 
tinuum breakdown on the surface properties of a $12 \mathrm{in}$. diameter, two-dimensional cylinder in a Mach 10 flow of argon at free stream Knudsen numbers from 0.002 to 0.25 . Their study found that as the Knudsen number increased, the level of agreement between CFD and DSMC diverged. A more recent study by Lofthouse et $a l^{4}$ examined the effects of velocity slip and temperature jump at the surface of a twodimensional, 12 in. diameter cylinder in Mach 10 and 25 flow of argon for the same free stream Knudsen numbers. In that study, it was found that the higher velocity did not increase the differences between CFD and DSMC. It was also observed that velocity slip and temperature jump boundary conditions improved the agreement between the two numerical methods. Another study by Lofthouse et al. ${ }^{6}$ investigated Mach 10 and 25 nitrogen flow over a two-dimensional cylinder. That study was again conducted over a range of Knudsen numbers from continuum to rarefied flow.

The purpose of this work is to accurately characterize the effects of continuum breakdown. This has to be performed by starting out with simple simulations and then adding complexity to determine individual effects on continuum breakdown. The work performed by Lofthouse et al. started this effort by characterizing breakdown over a twodimensional cylinder in a flow of argon and nitrogen. This was recently extended to flow of nitrogen over a 12 in. sphere for Mach 10, 25, and 45 flows to be able to include a geometry that is more representative of reentry vehicles. ${ }^{7}$ The present study will continue to examine the effects of continuum breakdown in a Mach 25 flow of reacting air over a 12 in. diameter sphere in flow regimes from continuum to rarefied gas. This adds the complexity of thermal and chemical nonequilibrium.

This paper will discuss the simulation procedures as well as the computational models used to perform the simulations. The paper will then discuss the surface properties predicted by CFD and DSMC in reacting air in several different flow regimes. A discussion of general trends observed in the drag, stagnation pressure, and stagnation heat flux follows. Finally, conclusions are discussed.

\section{NUMERICAL METHODS}

The DSMC simulations are performed using MONACO, a code developed at the University of Michigan by Boyd et al. ${ }^{8}$ MONACO is a parallel, unstructured, two-dimensional/threedimensional (2D/3D) DSMC code and it includes variable vibrational and rotational energy exchange models. The variable hard sphere (VHS) model ${ }^{2}$ is employed in this study. The final mesh used for each simulation is adapted by hand from previous simulations such that the cell size is of the same order as the local mean free path.

For this study, a hybrid mesh, one with both structured and unstructured cells, with cell stretching is utilized. A structured grid with cell stretching is employed along the fore body surface, while an unstructured mesh is used everywhere else in the flow field. This means the cell widths are adapted to be on the order of a mean free path, while the cell heights near the axis are stretched larger than the mean free path. This procedure creates a larger cell volume in this
TABLE I. Variable hard sphere temperature exponents.

\begin{tabular}{lccccc}
\hline \hline & $\mathrm{N}_{2}$ & $\mathrm{O}_{2}$ & $\mathrm{NO}$ & $\mathrm{N}$ & $\mathrm{O}$ \\
\hline $\mathrm{N}_{2}$ & 0.68 & 0.69 & 0.72 & 0.72 & 0.69 \\
$\mathrm{O}_{2}$ & 0.69 & 0.68 & 0.69 & 0.73 & 0.74 \\
$\mathrm{NO}$ & 0.72 & 0.69 & 0.70 & 0.76 & 0.73 \\
$\mathrm{~N}$ & 0.72 & 0.73 & 0.76 & 0.74 & 0.74 \\
$\mathrm{O}$ & 0.69 & 0.74 & 0.73 & 0.74 & 0.77 \\
\hline \hline
\end{tabular}

region so more particles can populate the cells near the stagnation point. This is important in axisymmetric simulations where it is difficult to obtain an appropriate number of particles per cell. ${ }^{9}$ This does not affect the simulation results because the primary flow gradients along the stagnation line are aligned with the cell widths, which are small enough to properly simulate the flow.

The CFD simulations are performed by solving the Navier-Stokes equations by use of the Michigan aerothermodynamic Navier-Stokes code LeMANS, developed at the University of Michigan for the simulation of hypersonic reacting flows. ${ }^{10,11}$ LeMANS is a parallel, unstructured 2D/3D, finite-volume CFD code. LeMANS has the ability to simulate gases in chemical, rotational, and vibrational nonequilibrium. LeMANS has three slip boundary conditions; ${ }^{4}$ for this study, Gökçen's slip boundary condition is utilized. A modified Steger-Warming flux vector splitting is employed to discretize the numerical fluxes between cells, which has low dissipation and is appropriate near boundary layers. A line implicit method is employed for the time integration. Even though LeMANS can handle unstructured meshes, all the simulations performed for this study are carried out using structured meshes. For each case, a grid convergence study is performed to determine the final mesh utilized.

It is necessary to ensure that the transport properties are the same in both CFD and DSMC. However, since these are simulations of multispecies flows, a mixing rule for the transport properties has to be utilized. There are two different models in the CFD method to calculate the transport properties of gas mixtures. The first utilizes Wilke's mixing rule, ${ }^{12}$ using the VHS viscosity model ${ }^{7}$ and Eucken's relation for thermal conductivity. The second utilizes Gupta's mixing rule, ${ }^{13}$ employing collision cross section data. For high temperature flows, it is suggested ${ }^{14,15}$ to utilize Gupta's mixing rule. Gupta's mixing rule calculates the transport properties from an approximation to the first-order Chapman-Enskog expression utilizing the collision cross sections. The collision integrals utilized in this study can be found in Wright et al. ${ }^{16}$

In order to ensure that the transport properties are being handled in the same way in both the DSMC and CFD methods, the viscosity collision integral is utilized to find VHS parameters for DSMC. A linear regression is performed on the logarithm of the viscosity collision integral versus the logarithm of the temperature to calculate the VHS $\omega$ values, which are given in Table I. 


\section{A. Vibrational relaxation}

There are two models for the exchange of vibrational and translational energy in DSMC; the first is a phenomenological model as described by Boyd. ${ }^{17}$ The probability of an inelastic collision where vibrational energy is exchanged with translational energy is proportional to the inverse of the vibrational relaxation time. This probability is the average over the instantaneous probabilities as shown in Eq. (3)

$$
P=\frac{1}{\tau_{v} \nu}=\int_{0}^{\infty} \phi(g) f(g) d g,
$$

where $\phi(\mathrm{g})$ is the instantaneous probability for a given relative velocity and relative velocity distribution function.

When the instantaneous probability is integrated over all collisions, it should match the average probability calculated from theory; however, it was found that they do not match. It is thought that the probabilities do not match due to the method of steepest descent required to find the instantaneous probability. It has been found that the probability can better correspond with theory by multiplying by a simple factor that is dependent on the maximum temperature. ${ }^{6}$

Unfortunately, this method does not work for multiple species and in fact was only employed for $\mathrm{N}_{2}-\mathrm{N}_{2}$ vibrational relaxation. ${ }^{7}$ For multiple species, a more elegant solution has been included in the DSMC method to be able to obtain the proper vibrational relaxation rate. The probability for each collision class and each cell is calculated using Eq. (3). The vibrational relaxation time is calculated by using the Landau-Teller model with correlated experimental data from Millikan and White ${ }^{18}$ and a correction proposed by Park. ${ }^{19} \mathrm{~A}$ factor proposed by Gimelshein et al. ${ }^{20}$ is needed to be able to utilize this relaxation time in a particle simulation as shown in Eq. (4)

$$
\tau_{\text {part }}=\frac{\tau_{\text {cont }}}{1+\frac{0.5 \zeta^{2} \exp \left(\theta_{v} / T\right)}{4-2 \omega}} .
$$

This equation can be employed as long as the vibrational temperature is less than the translational temperature, which is the case for hypersonic flows. Note that it also works if the vibrational temperature is slightly greater than the translational temperature.

This model implemented in DSMC was shown by Deschenes et $_{\text {al. }}{ }^{21}$ to match the vibrational relaxation in CFD for nitrogen. Since this model has only been tested with one species, a heat bath of five species air is run with the cell based model and the phenomenological model in DSMC and compared to CFD. The heat bath is started with a translational temperature of $15000 \mathrm{~K}$, while rotational and vibrational temperatures are started at $10000 \mathrm{~K}$. From the results of this test, which are displayed in Fig. 1, it can be seen that the cell based method is in better agreement with CFD than the variable vibration probability.

\section{B. Chemistry models}

In hypersonic flows, it is common to have chemical reactions around the reentry vehicle. This study is concerned

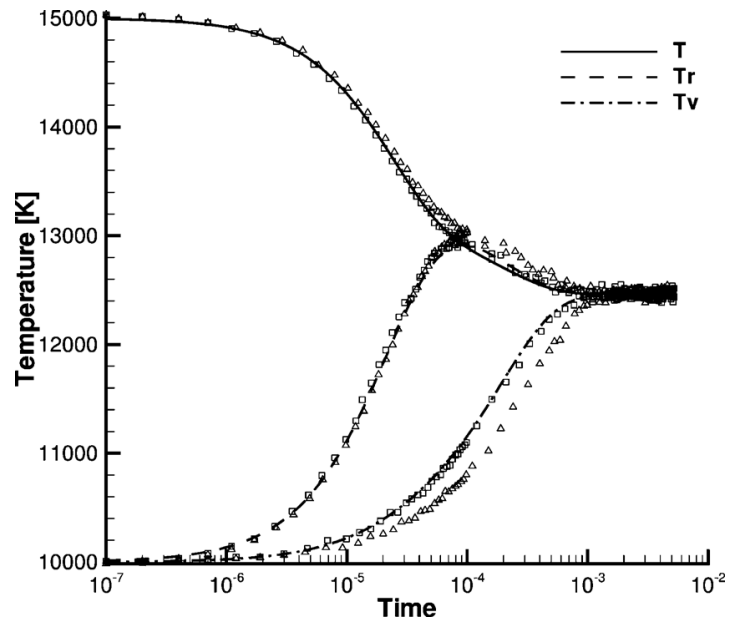

FIG. 1. Comparison of thermal relaxation process as predicted by DSMC and CFD in five species air; squares represent DSMC with the cell based model and triangles represent DSMC and lines represent CFD.

with simulations of a reentry flow in five species reacting air. Thus, it is important to make sure that the reaction rates calculated in the CFD and DSMC methods are performed in a similar manner. This is done to ensure that any differences seen between the two codes are not due to different reaction rates. The following sections highlight differences in how chemically reacting flow is handled in the two methods and what is done to make both numerical methods behave in a similar manner.

\section{Chemical equilibrium}

One of the major differences between the CFD and DSMC methods is how chemical equilibrium is handled. Thus, the first step is making sure the forward and backward reaction rates, and hence the equilibrium constant, are all calculated in a similar manner. First, one needs to understand what happens in the DSMC method.

In the DSMC method, a collision pair is selected and the probability of reaction is compared to a random number. If the probability is greater than the random number, then a reaction occurs. Once a reaction occurs, a Borgnakke-Larsen model is applied to distribute the energy to the available energy modes. There are two models available in MONACO for chemistry: the total collision energy (TCE) model and the vibrationally favored dissociation (VFD) model. ${ }^{22}$ The VFD model is employed in the DSMC method for this study and a qualitatively similar model is used in the CFD method, as discussed in greater detail in Sec. II B 3. For both the TCE and VFD models, the reaction rate coefficient, forward or backward, must be in modified Arrhenius form. The probability of reaction is found by integration over the equilibrium Boltzmann distribution function as shown in Eq. (5)

$$
\begin{aligned}
k_{f}(T)= & a_{f} T^{\eta_{f}} \exp \left(\frac{-\varepsilon_{f}}{k_{\text {BOLTZ } T}}\right) \\
= & \langle\sigma g\rangle \int_{\varepsilon_{f}}^{\infty} P_{c}\left(\varepsilon_{c}\right) \int_{\varepsilon_{v}=0}^{\infty} P_{v}\left(\varepsilon_{v}\right) f_{B}\left(\varepsilon_{v}\right) \\
& \times f_{B}\left(\varepsilon_{c}-\varepsilon_{v}\right) d \varepsilon_{v} d \varepsilon_{c},
\end{aligned}
$$


where $f_{B}$ is the equilibrium Boltzmann distribution for energy. Since the reaction rate has the modified Arrhenius form, the reaction probability for the VFD model is found to be

$$
P_{\mathrm{VFD}}=A\left(\frac{\varepsilon_{v}}{\varepsilon_{c}}\right)^{\phi} \frac{\left(\varepsilon_{c}-\varepsilon_{f}\right)^{\psi}}{\left(\varepsilon_{c}\right)^{\chi}},
$$

where $A$ is a complicated constant not reproduced here, $\phi$ is a constant dependent on the species, ${ }^{23} \psi=\eta+0.5+\zeta$, and $\chi=1+\zeta-\omega$. If $\phi$ is set to zero, the TCE model is recovered from the VFD model.

In order to simulate backward reactions with the VFD model, the rate coefficient must be in the modified Arrhenius form. The backward rate coefficients are calculated using the forward rate coefficients and the equilibrium constant, as shown in Eq. (7)

$$
k_{b}(T)=\frac{k_{f}(T)}{K_{e}(T)}=a_{b} T^{\eta_{b}} \exp \left(\frac{-\varepsilon_{b}}{k_{\mathrm{BOLTZ}} T}\right) .
$$

Typically, in the DSMC method, the backward rates are fit to a modified Arrhenius form over a specified temperature range. ${ }^{24}$ This can cause problems if a simulation goes outside this temperature range and all the fits need to be redone. It is common in the CFD method to use a line fit proposed by Park $^{19}$ to find the equilibrium constant, as given in Eq. (8). This equilibrium constant is then applied to find the backward reaction rate coefficient

$$
\begin{aligned}
K_{e}(T)= & \exp \left[A_{1}\left(\frac{10000}{T}\right)+A_{2}+A_{3} \ln \left(\frac{T}{10000}\right)\right. \\
& \left.+A_{4}\left(\frac{T}{10000}\right)+A_{5}\left(\frac{T}{10000}\right)^{2}\right] .
\end{aligned}
$$

The constants $A_{i}$ are dependent on the number density as given by Park. ${ }^{19}$ The constants are found using an interpolation method if the number density is within the range of the data. If the number density falls outside that range, the constants at the highest or lowest points are used accordingly. For DSMC, instead of performing a fit over a limited temperature range, it would be desirable to evaluate the equilibrium constant and then calculate the backward reaction rate coefficients. This can be done by a method suggested by Boyd ${ }^{22}$ which utilizes the equilibrium constant and maintains the modified Arrhenius form required by the DSMC chemistry model. The backward reaction rate coefficient is found by taking the forward reaction rate coefficient [Eq. (5)] and, substituting into Eq. (7), one can solve for the backward rate constant as shown in Eq. (9)

$$
a_{b}=\frac{a_{f}}{K_{e}(T)} \exp \left(\frac{-\varepsilon_{f}}{k_{\mathrm{BOLTZ}} T}\right) .
$$

This can now be substituted into the modified Arrhenius form and the probability for the backward reaction can be found in the same way as described for the forward reaction. In this method, the forward and backward reaction rate coefficients in DSMC are calculated in a similar manner as CFD, despite the vast differences in the way the chemical reactions are handled in both numerical methods.

\section{Three-temperature model}

In Sec. II B 1, the methods for calculating the forward and backward rate coefficients were given, which are utilized in both CFD and DSMC methods. The temperature that is employed in the CFD calculations is Park's two-temperature model, ${ }^{19}$ given in Eq. (10)

$$
T_{P}=T_{\mathrm{tr}}^{a} T_{\mathrm{ve}}^{b},
$$

where $a$ and $b$ are constants that must sum to $1, T_{\text {tr }}$ is the translation-rotational temperature, and $T_{\mathrm{ve}}$ is the vibrationalelectron-electronic temperature. The reaction rates are affected by the level of thermal nonequilibrium present in the flow; the two-temperature model is attempting to include the effects of thermal nonequilibrium. By including the vibrational temperature, this model aims to account for the fact that vibrationally excited particles are more likely to dissociate. Values for $a$ and $b$ can vary, but there are two typical sets utilized: $a=b=0.5$ or $a=0.7$ and $b=0.3$. For the backward reaction rate, and hence the equilibrium constant, the temperature utilized is the translation-rotational temperature; therefore $a=1$, while $b=0$. This is the same for exchange reactions.

In a previous study, a separate rotational energy equation was included into LeMANS, ${ }^{7}$ giving it the ability to simulate rotational nonequilibrium. Since there is now a separate rotational temperature, it has to be included in the reaction rates. There can be rotational thermal nonequilibrium; therefore, the temperature employed in the rate calculation is now composed of three temperatures. The need for a so-called three-temperature model is now being recognized as a necessity in chemistry modeling by Park. ${ }^{25}$ In this work, a phenomenological temperature model is created based on the two-temperature model

$$
T_{P}=T_{t}^{a} T_{r}^{b} T_{\mathrm{ve}}^{c},
$$

where the $a, b$, and $c$ values again must sum to $1, T_{t}$ is the translational temperature, $T_{r}$ is the rotational temperature, and $T_{\mathrm{ve}}$ is the vibrational-electron-electronic temperature. There has been research on finding the reaction rates that work best with the two-temperature model. ${ }^{26-28}$ This work cannot produce reaction rates that best match this temperature model, so a method of employing the degrees of freedom is devised. The same emphasis on the vibrational temperature is retained, so $c$ is kept at 0.5 . The remaining 0.5 is split over translational and rotational temperatures by the number of degrees of freedom, so that $a$ is set to 0.3 and $b$ is set to 0.2 .

For the backward and exchange reactions, the same method is utilized. The temperature utilized for these reactions is the translational temperature. As a result, the value of $a$ is set to 1.0 and both $b$ and $c$ are set to 0 . Utilizing this model puts a stronger emphasis on molecules with higher vibrational and rotational energies, allowing the CFD method to include thermal nonequilibrium effects on the reaction rates. It should be noted that when the flow is in rotational equilibrium, this phenomenological three-temperature model reduces to the two-temperature model. 

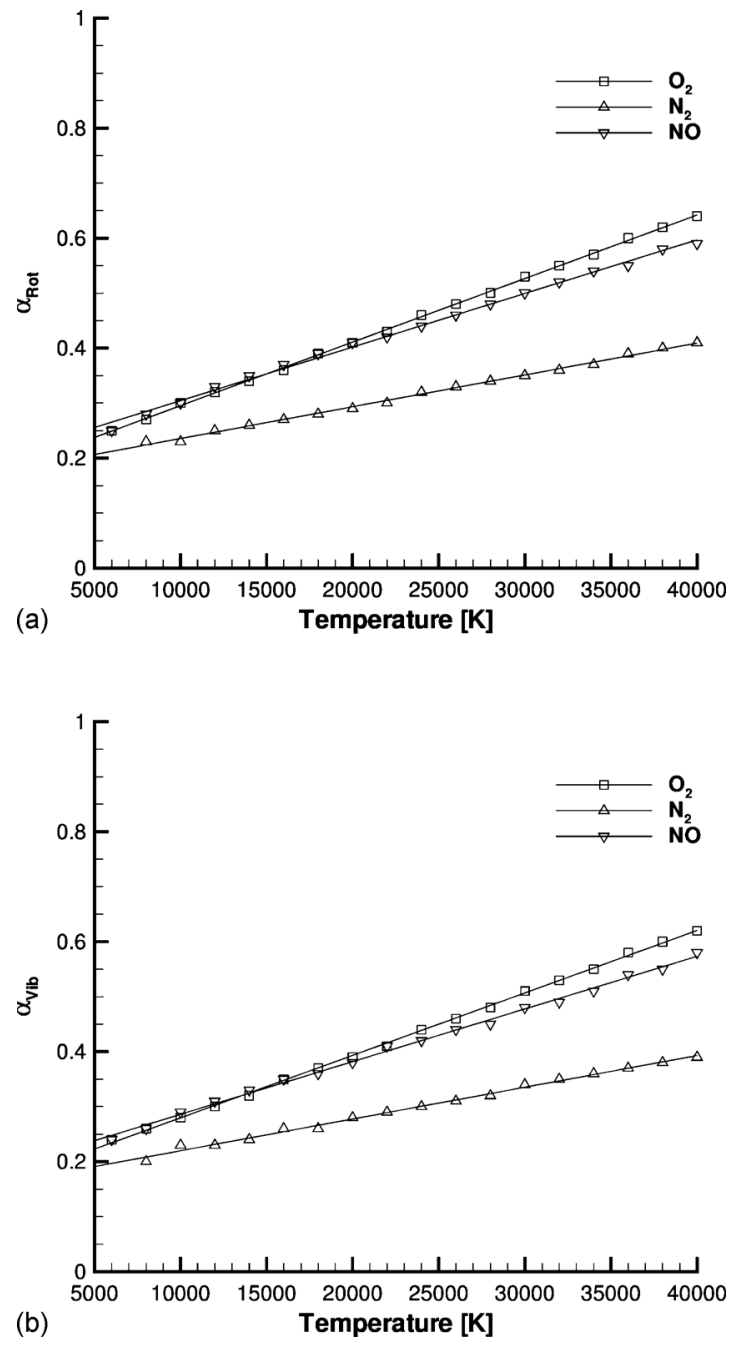

FIG. 2. (a) Rotational and (b) vibrational preferential dissociation alpha as a function of temperature; symbols are data from DSMC method and lines represent the line fits utilized.

\section{Preferential dissociation}

In a further attempt to have the CFD method behave more like the DSMC method with the VFD model, a preferential dissociation model is included in the CFD method. Without the preferential model, it is assumed that the molecules are destroyed or created at the average vibrational energy of the cell. In the preferential model, it is assumed that the molecules are destroyed or created at a higher vibrational energy. This preferential dissociation model is implemented in the source term for the vibrational energy equation, the source term is given in Eq. (12).

$$
S=\dot{\omega}_{s}\left(\alpha_{s} D_{s}\right),
$$

where $D_{s}$ is the dissociation potential for a given species, $\dot{\omega}_{s}$ is the species conservation source term, and $\alpha_{s}$ is the fraction of the dissociation potential that is due to vibrational energy. The $\alpha_{s}$ value is usually set to 0.3 . While this model is typically only applied to vibrational preferential dissociation, in this work it is also applied to rotational preferential dissociation. To be able to have the preferential dissociation model in the CFD method match the DSMC method with the VFD
TABLE II. Reaction rates employed in DSMC and CFD.

\begin{tabular}{lcrc}
\hline \hline Reaction & $\begin{array}{c}\mathrm{a} \\
\left(\mathrm{m}^{3} / \mathrm{molecule} / \mathrm{s}\right)\end{array}$ & $\eta$ & $\begin{array}{c}\varepsilon / k_{\mathrm{BOLTZ}} \\
(\mathrm{K})\end{array}$ \\
\hline $\begin{array}{l}\text { Dissociation } \\
\mathrm{N}_{2}+M \rightleftharpoons \mathrm{N}+\mathrm{N}+M\left(M=\mathrm{N}_{2}, \mathrm{O}_{2}, \mathrm{NO}\right)\end{array}$ & $1.162 \times 10^{-8}$ & -1.6 & 113200 \\
$\mathrm{~N}_{2}+M \rightleftharpoons \mathrm{N}+\mathrm{N}+M(M=\mathrm{N}, \mathrm{O})$ & $4.980 \times 10^{-8}$ & -1.6 & 113200 \\
$\mathrm{O}_{2}+M \rightleftharpoons \mathrm{O}+\mathrm{O}+M\left(M=\mathrm{N}_{2}, \mathrm{O}_{2}, \mathrm{NO}\right)$ & $3.321 \times 10^{-9}$ & -1.5 & 59400 \\
$\mathrm{O}_{2}+M \rightleftharpoons \mathrm{O}+\mathrm{O}+M(M=\mathrm{N}, \mathrm{O})$ & $1.660 \times 10^{-8}$ & -1.5 & 59400 \\
$\mathrm{NO}+M \rightleftharpoons \mathrm{N}+\mathrm{O}+M\left(M=\mathrm{N}_{2}, \mathrm{O}_{2}, \mathrm{NO}\right)$ & $8.302 \times 10^{-15}$ & 0.0 & 75500 \\
$\mathrm{NO}+M \rightleftharpoons \mathrm{N}+\mathrm{O}+M(M=\mathrm{N}, \mathrm{O})$ & $1.826 \times 10^{-13}$ & 0.0 & 75500 \\
& & & \\
Exchange & & & \\
$\mathrm{NO}+\mathrm{O} \rightleftharpoons \mathrm{O}+\mathrm{N}$ & $1.395 \times 10^{-17}$ & 0.0 & 19450 \\
$\mathrm{~N}_{2}+\mathrm{O} \rightleftharpoons \mathrm{NO}+\mathrm{N}$ & $1.063 \times 10^{-12}$ & -1.0 & 38400 \\
\hline \hline
\end{tabular}

model, data from the DSMC method are utilized to find new $\alpha_{s}$ values. The amount of rotational and vibrational energy lost per reaction is found from DSMC simulations of heat baths and the $\alpha_{s}$ values are given in Fig. 2.

From this figure, it can be seen that at lower temperatures, between 10000 and $15000 \mathrm{~K}, \alpha_{s}$ is around 0.3 , making the original CFD assumption fairly accurate. However, the values grow higher as the temperature rises. In the figure, line fits to the DSMC data are also shown. These line fits are given in Eqs. (13)-(15)

$$
\begin{aligned}
& \alpha_{r, \mathrm{~N}_{2}}=5.784 \times 10^{-6} T+0.178, \\
& \alpha_{v, \mathrm{~N}_{2}}=5.772 \times 10^{-6} T+0.162, \\
& \alpha_{r, \mathrm{O}_{2}}=1.156 \times 10^{-5} T+0.179, \\
& \alpha_{v, \mathrm{O}_{2}}=1.136 \times 10^{-5} T+0.166, \\
& \alpha_{r, \mathrm{NO}}=9.752 \times 10^{-6} T+0.207, \\
& \alpha_{v, \mathrm{NO}}=9.582 \times 10^{-5} T+0.190 .
\end{aligned}
$$

These equations are implemented into the CFD method to better match the VFD model in the DSMC method.

To test this model, a heat bath simulation is performed. The reaction rates utilized in this study are given in Table II. The test case considers air starting at a temperature of $15000 \mathrm{~K}$.

Figure 3(a) gives the mole fraction profiles over time for DSMC, the original model and the new chemical equilibrium model, and CFD. It can be seen that both DSMC models compare very well with each other and CFD for a simple five species air model. The largest difference is observed to occur in nitric oxide, where the peak difference is approximately $10 \%$ between CFD and DSMC. The temperature profiles from the test case are given in Fig. 3(b). From this figure, it is seen that the two methods predict similar temperature profiles, with a maximum error of approximately $10 \%$ between CFD and DSMC. It should be noted that there is less than $1 \%$ difference between the two DSMC implementations. 

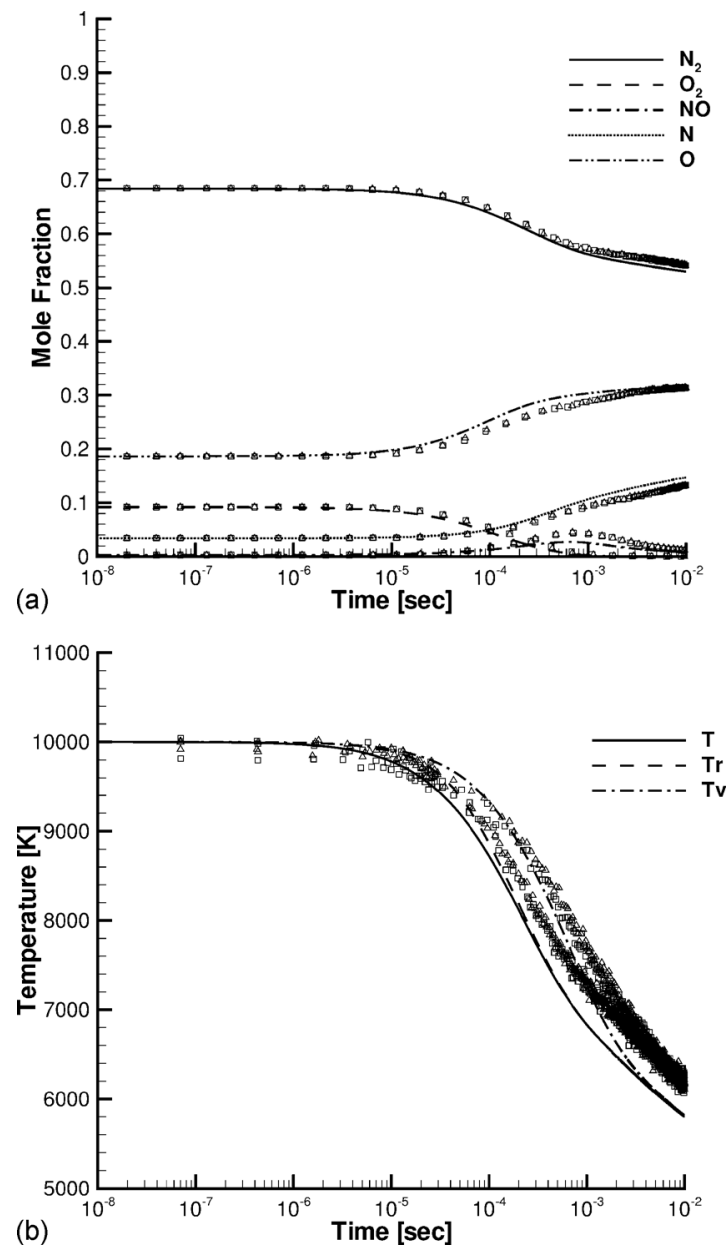

FIG. 3. Comparison of chemical reaction process as predicted by DSMC and CFD in air; triangles represent DSMC with Park's equilibrium, squares represent DSMC, and lines represent CFD.

From the profiles of mole fraction and temperature, it is clear there are still some differences between CFD and DSMC. To understand these differences, particle data are extracted from the heat bath test case and the probability distribution functions are found. The probability distribution functions are computed by placing particles in bins; usually, this is accomplished by specifying how many bins are desired and then sorting the particles into the bins. In this study, a different approach is utilized, where the number of particles per bin is specified and the width of the bin is variable. $^{29}$ The probability is the number of particles in the bin over the width of the bin and normalized by the total number of particles, as given in Eq. (16)

$$
f(x) d x=\frac{1}{n} \frac{\text { No. of } X_{i} \text { in same bin as } x}{\text { Width of bin containing } x} d x
$$

where $n$ is the total number of particles, the numerator is the number of particles in the bin, and the denominator is the width of the bin. To make comparisons to CFD, the particle velocity distributions are compared to the Boltzmann distribution. The Boltzmann distribution ${ }^{1}$ for all internal energies is given below
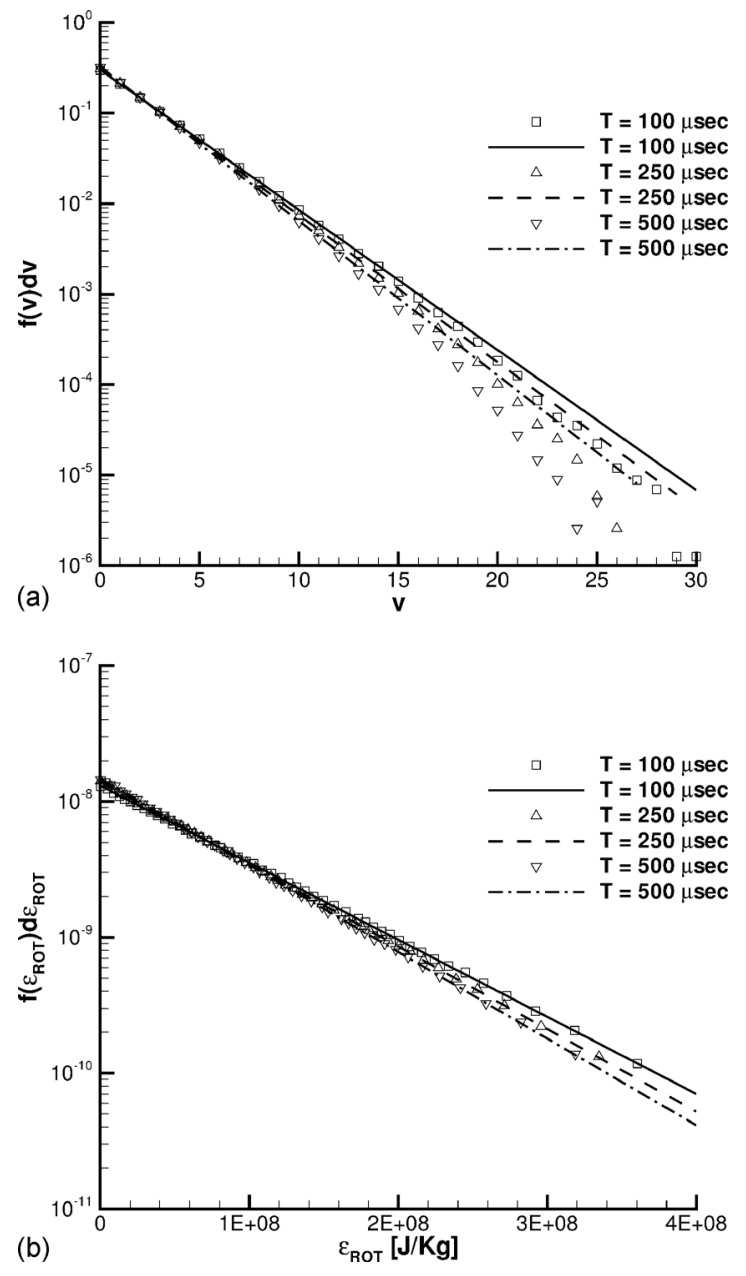

FIG. 4. (a) Vibrational and (b) rotational probability distribution functions; symbols represent particle data, while lines represent equilibrium Boltzmann's distribution.

$$
\frac{N_{j}^{*}}{N}=\frac{g_{j} \exp \left(-\frac{e_{j}}{k_{\mathrm{BOLTZ}} T}\right)}{Q},
$$

where $e_{j}$ is the energy, $T$ is the temperature associated with the energy mode, $g_{j}$ is the degeneracy, and $Q$ is the partition function of the energy mode. This can be utilized to obtain the Boltzmann distribution for either rotational or vibrational energy. It should be noted that the rotational energy utilizes a continuous version of the Boltzmann distribution, ${ }^{30}$ given Eq. (18)

$$
f(\varepsilon) d \varepsilon=\frac{1}{\Gamma(\zeta / 2)}\left(\frac{\varepsilon}{k_{\mathrm{BOLTZ}} T}\right)^{\zeta / 2-1} \exp \left(\frac{-\varepsilon}{k_{\mathrm{BOLTZ}} T}\right) \frac{d \varepsilon}{k_{\mathrm{BOLTZ}} T},
$$

where $\varepsilon$ is the rotational energy and $\zeta$ is the number of degrees of freedom associated with the rotational energy mode.

Even though this is a heat bath test case, there is still significant vibrational nonequilibrium as can be seen in Fig. 4(a). This figure gives the distribution function along with an equilibrium Boltzmann distribution. The vibrational nonequilibrium is caused by chemical reactions depleting higher 
TABLE III. Simulated flow regimes.

\begin{tabular}{lccc}
\hline \hline & $\begin{array}{c}\text { Mass density } \\
\left(\mathrm{kg} / \mathrm{m}^{3}\right)\end{array}$ & $\begin{array}{c}\text { Number density } \\
\left(\text { particles } / \mathrm{m}^{3}\right)\end{array}$ & $\begin{array}{c}\text { Mean free path } \\
(\mathrm{m})\end{array}$ \\
\hline 0.002 & $1.007 \times 10^{-4}$ & $2.103 \times 10^{21}$ & $6.096 \times 10^{-4}$ \\
0.01 & $2.014 \times 10^{-5}$ & $4.206 \times 10^{20}$ & $3.048 \times 10^{-3}$ \\
0.05 & $3.987 \times 10^{-6}$ & $8.325 \times 10^{19}$ & $1.524 \times 10^{-2}$ \\
0.25 & $8.057 \times 10^{-7}$ & $1.680 \times 10^{19}$ & $7.620 \times 10^{-2}$ \\
\hline \hline
\end{tabular}

vibrational energy states faster than the vibrational energy distribution can equilibrate. Notice that the same behavior is not true for rotational energy because rotational relaxation is faster than chemical reactions. Since the vibrational distribution function has a direct impact on the reaction rates [see Eq. (5)], the vibrational nonequilibrium is the cause of the differences seen in the previous heat bath results. Since the CFD method is not able to simulate this vibrational nonequilibrium, it will not be able to match the reaction rates calculated in the DSMC methods.

\section{RESULTS}

This study examines continuum breakdown in Mach 25 flows of reacting air over a 12 in. diameter sphere. The free stream temperature is $200 \mathrm{~K}$, giving a free stream velocity of $7108 \mathrm{~m} / \mathrm{s}$ for air. The surface of the sphere has a fixed temperature of $1500 \mathrm{~K}$. The density of the free stream is varied to change the global Knudsen number of the flow from continuum to a rarefied gas as given in Table III. The Knudsen number is calculated using the diameter of the sphere as the characteristic length and the hard sphere model to calculate the mean free path.

The purpose of this study is to compare surface properties predicted by DSMC and CFD simulations, heat flux, pressure, and shear stress to examine any differences occurring due to continuum breakdown. Additionally, the integrated drag and the maximum heat flux are also compared from DSMC and CFD. The maximum heat flux is found by averaging the heat flux over the surface of the sphere within the first degree of the stagnation point. This is done to be able to determine the maximum heat flux predicted by DSMC, which can have some random noise in the results. Since the CFD solutions are smooth, there is no need for averaging and the maximum heat flux is found by finding the maximum value of the heat flux on the surface of the sphere.

The results that are presented in this paper for the surface aerothermodynamic properties are given as nondimensionalized coefficients which are defined by Eqs. (19)-(21)

$$
\begin{gathered}
C_{p}=\frac{p-p_{\infty}}{\frac{1}{2} \rho_{\infty} U_{\infty}^{2}}, \\
C_{\tau}=\frac{\tau}{\frac{1}{2} \rho_{\infty} U_{\infty}^{2}},
\end{gathered}
$$

TABLE IV. Integrated drag (N) (\% difference) from DSMC and CFD at Mach 25 in reacting air.

\begin{tabular}{cccc}
\hline \hline $\mathrm{Kn}_{\infty}$ & DSMC & CFD (no slip) & CFD (slip) \\
\hline 0.002 & 178 & $175(-1.69 \%)$ & $174(-2.25 \%)$ \\
0.01 & 40.8 & $41.0(0.49 \%)$ & $40.3(-1.22 \%)$ \\
0.05 & 10.1 & $11.8(16.8 \%)$ & $9.79(-3.07 \%)$ \\
0.25 & 2.53 & $4.80(89.9 \%)$ & $2.58(1.98 \%)$ \\
\hline \hline
\end{tabular}

$$
C_{q}=\frac{q}{\frac{1}{2} \rho_{\infty} U_{\infty}^{3}} .
$$

The surface aerothermodynamic properties are plotted against the surface angle $\phi$, which is measured from the stagnation point.

Five species, reacting air involves 17 reactions, which includes dissociation and exchange reactions; the reaction rates are given in Table II. The integrated drag, peak heat flux, and percent difference between DSMC and CFD are given in Tables IV and V, respectively. Since DSMC is a particle method that works in both the continuum regime and the rarefied regime, it is assumed that the DSMC results are more accurate, so the percent difference is calculated using the DSMC result as the basis.

From these tables, it can be seen that the agreement between CFD and DSMC diverges with growing global Knudsen number. In Secs. III A-III D, the surface properties and gradient length local Knudsen number are discussed in more detail for each case.

\section{A. $\mathrm{Kn}_{\infty}=0.002$}

Given the global Knudsen number, the flow is expected to be in the continuum regime. However, there are regions of local continuum breakdown in the shock and wake regions as shown in Fig. 5(a). There is a larger area of continuum breakdown observed in DSMC over CFD in both the shock region and in the wake region behind the sphere.

The surface pressure coefficient is given in Fig. 5(b), along with the surface profile of $\mathrm{Kn}_{G L L}$. Notice that $\mathrm{Kn}_{G L L}$ is above 0.05 for nearly the entire surface, indicating the flow is considered to be in continuum breakdown. Despite the continuum breakdown at the surface, the pressures predicted by DSMC and CFD match very well. The surface heat flux predicted by CFD compares well with DSMC over the fore body of the sphere, as shown in Fig. 6(a). The divergence in the heat flux between the two numerical methods may be caused by the breakdown on the surface, especially over the

TABLE V. Peak heating $\left(\mathrm{W} / \mathrm{m}^{2}\right)$ (\% difference) from DSMC and CFD at Mach 25 in reacting air.

\begin{tabular}{lccc}
\hline \hline $\mathrm{Kn}_{\infty}$ & DSMC & CFD (no slip) & CFD (slip) \\
\hline 0.002 & $9.30 \times 10^{5}$ & $9.46 \times 10^{5}(1.63 \%)$ & $9.26 \times 10^{5}(-0.46 \%)$ \\
0.01 & $8.04 \times 10^{5}$ & $8.98 \times 10^{5}(11.7 \%)$ & $8.42 \times 10^{5}(4.72 \%)$ \\
0.05 & $3.54 \times 10^{5}$ & $4.44 \times 10^{5}(25.5 \%)$ & $3.70 \times 10^{5}(4.60 \%)$ \\
0.25 & $1.05 \times 10^{5}$ & $1.72 \times 10^{5}(63.5 \%)$ & $8.55 \times 10^{4}(-18.7 \%)$ \\
\hline \hline
\end{tabular}



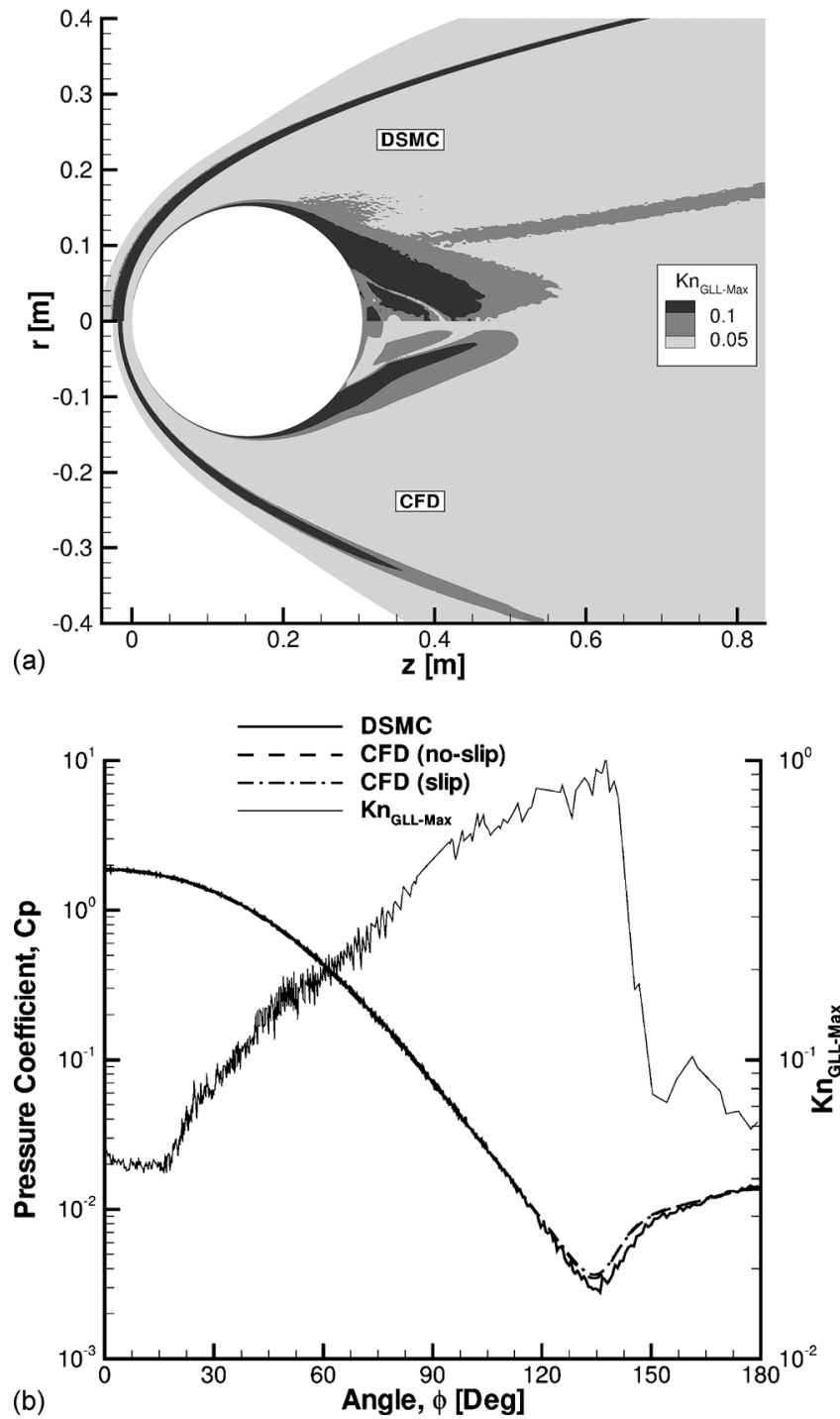

FIG. 5. $\mathrm{Kn}_{\infty}=0.002, \mathrm{Kn}_{G L L}$ contours, surface pressure (left axis), and $\mathrm{Kn}_{G L L}$ surface profile (right axis) on a sphere in a Mach 25 flow of reacting air.

aft of the sphere. The shear stress over the surface of the sphere is given in Fig. 6(b). The shear stress prediction given by CFD compares well with DSMC over the surface, with only slight disagreement in the aft of the sphere.

To determine the level of chemistry occurring in the flow, the mass fractions of each species are given in Fig. 7(a). It can be seen that in DSMC, trace species diffuse out from behind the shock. This phenomenon can be seen by the discrepancies between CFD and DSMC in mass fraction for nitric oxide, atomic oxygen, and atomic nitrogen that develop in front of the shock. It is unclear if this is a physical phenomenon or numerical problems in DSMC dealing with trace species. From the figure, it can be seen that the mass fractions predicted by the two methods compare well; the only noticeable difference is in the mass fraction for nitric oxide. The temperature and gradient length local Knudsen number profiles along the stagnation streamline are given in Fig. 7(b). It can be observed that the translational temperature matches well between the two methods. However, the rotational and vibrational temperatures do not match as well.
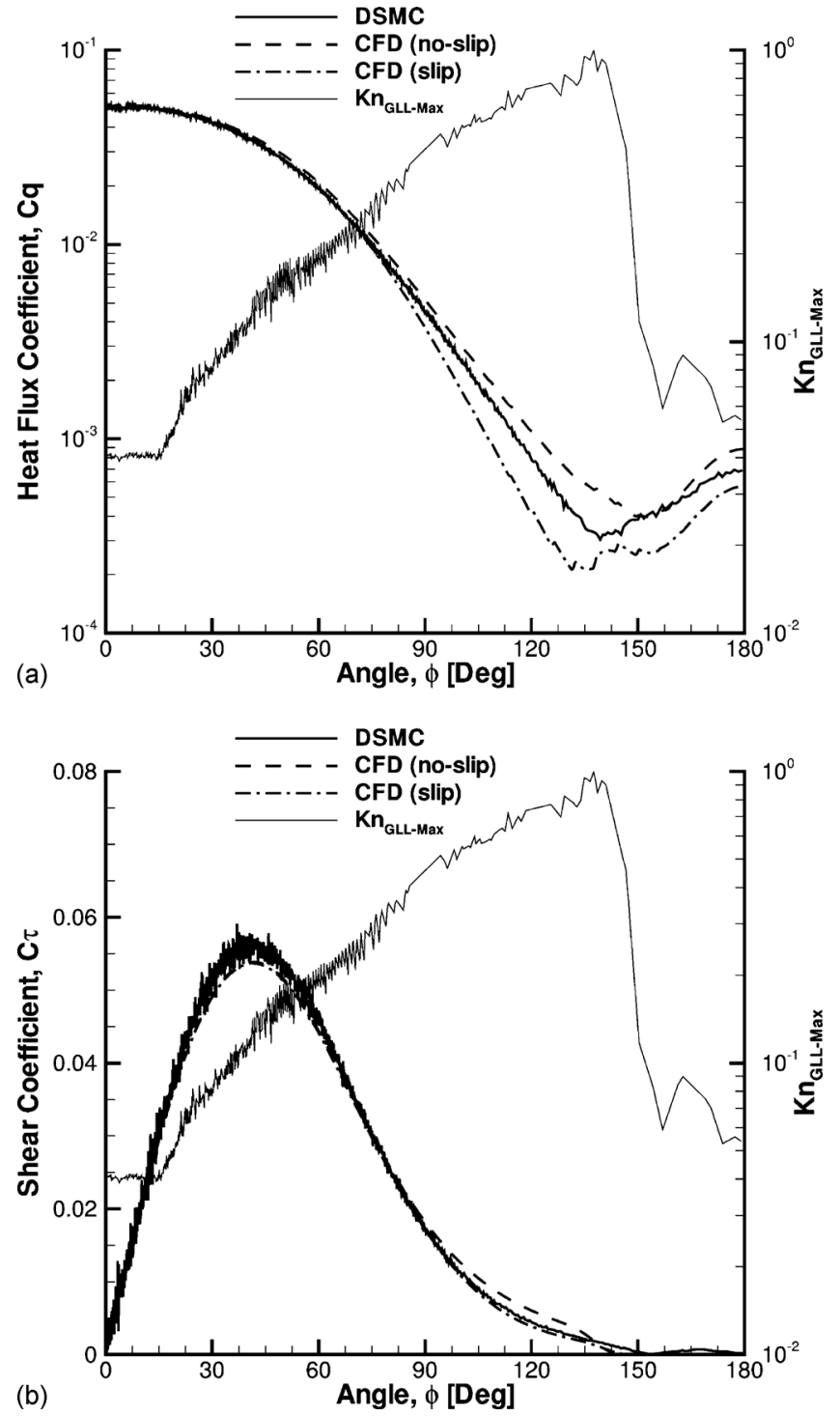

FIG. 6. $\mathrm{Kn}_{\infty}=0.002$, surface heat flux (left axis), surface shear stress (left axis), and $\mathrm{Kn}_{G L L}$ surface profile (right axis) on a sphere in a Mach 25 flow of reacting air.

The overprediction of rotational and vibrational temperatures by CFD as compared to DSMC is an expected result, caused by thermal nonequilibrium. ${ }^{7}$ From the profile of $\mathrm{Kn}_{G L L}$, it is seen that the flow is expected to be in the continuum regime as it approaches the wall.

\section{B. $K \mathrm{n}_{\infty}=0.01$}

At a global Knudsen number of 0.01, the traditional limit for accurate CFD simulations, there is significant breakdown in the shock, boundary layer, and the wake regions of the flow, as seen in Fig. 8(a). At this condition, the amount of continuum breakdown is larger in DSMC than in CFD. Notice that the flow is in continuum breakdown all the way from the shock to the surface of the sphere near the stagnation streamline.

The surface pressures computed from the CFD method, with or without slip boundary conditions, compare well with DSMC in the fore body of the sphere, as shown in Fig. 8(b). 


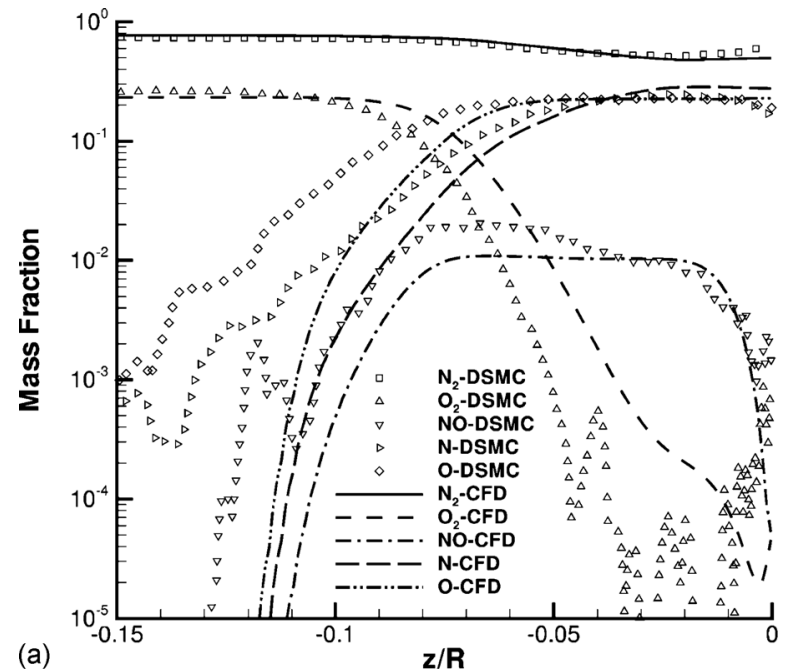

$\mathbf{z} / \mathbf{R}$

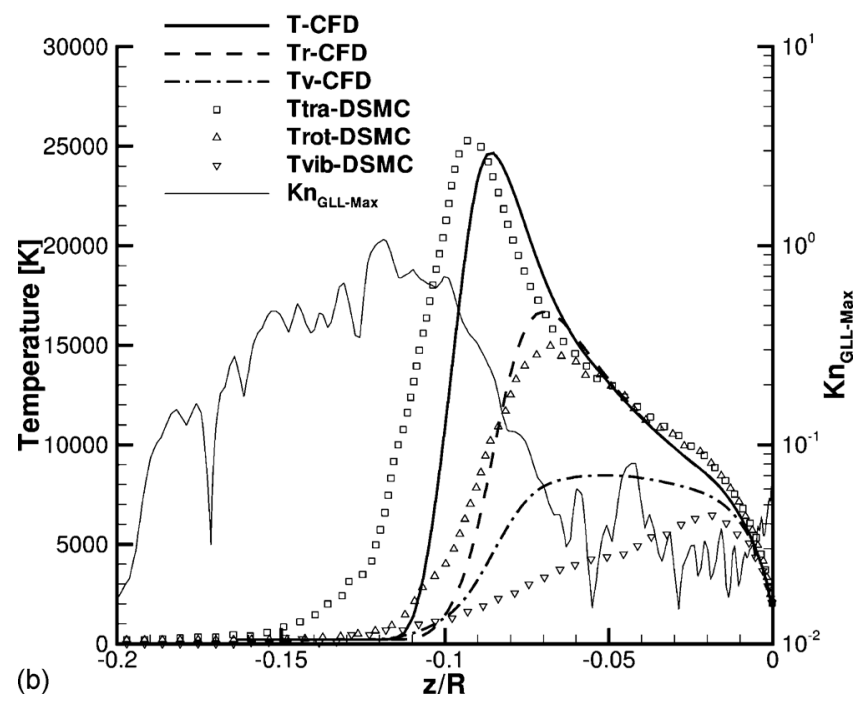

FIG. 7. $\mathrm{Kn}_{\infty}=0.002$, mass fraction (left axis) temperature (left axis), and $\mathrm{Kn}_{G L L}$ (right axis) profiles along the stagnation streamline in a Mach 25 flow of reacting air.

However, there is some discrepancy in pressure between CFD and DSMC methods in the aft of the sphere, where the level of breakdown is the greatest. It is interesting to note that the no-slip boundary conditions give the better agreement with DSMC than with slip boundary conditions. This figure also includes the surface profile of the gradient length local Knudsen number, which shows the flow over the entire surface is in continuum breakdown. The heat flux predicted by CFD with no slip is larger than DSMC over the entire surface, as can be seen in Fig. 9(a). An improvement is seen if slip boundary conditions are employed in the CFD method. At this Knudsen number, the CFD method, with and with out slip boundary conditions, underpredicts the shear stress near the location of the maximum, as shown in Fig. 9(b). It can also be seen that the CFD method overpredicts DSMC over the aft of the sphere. It is also interesting to note that the CFD method predicts that the peak shear stress occurs slightly later on the surface of the sphere.

To be able to determine the level of chemistry occurring in the flow, the mass fractions of each species are given in
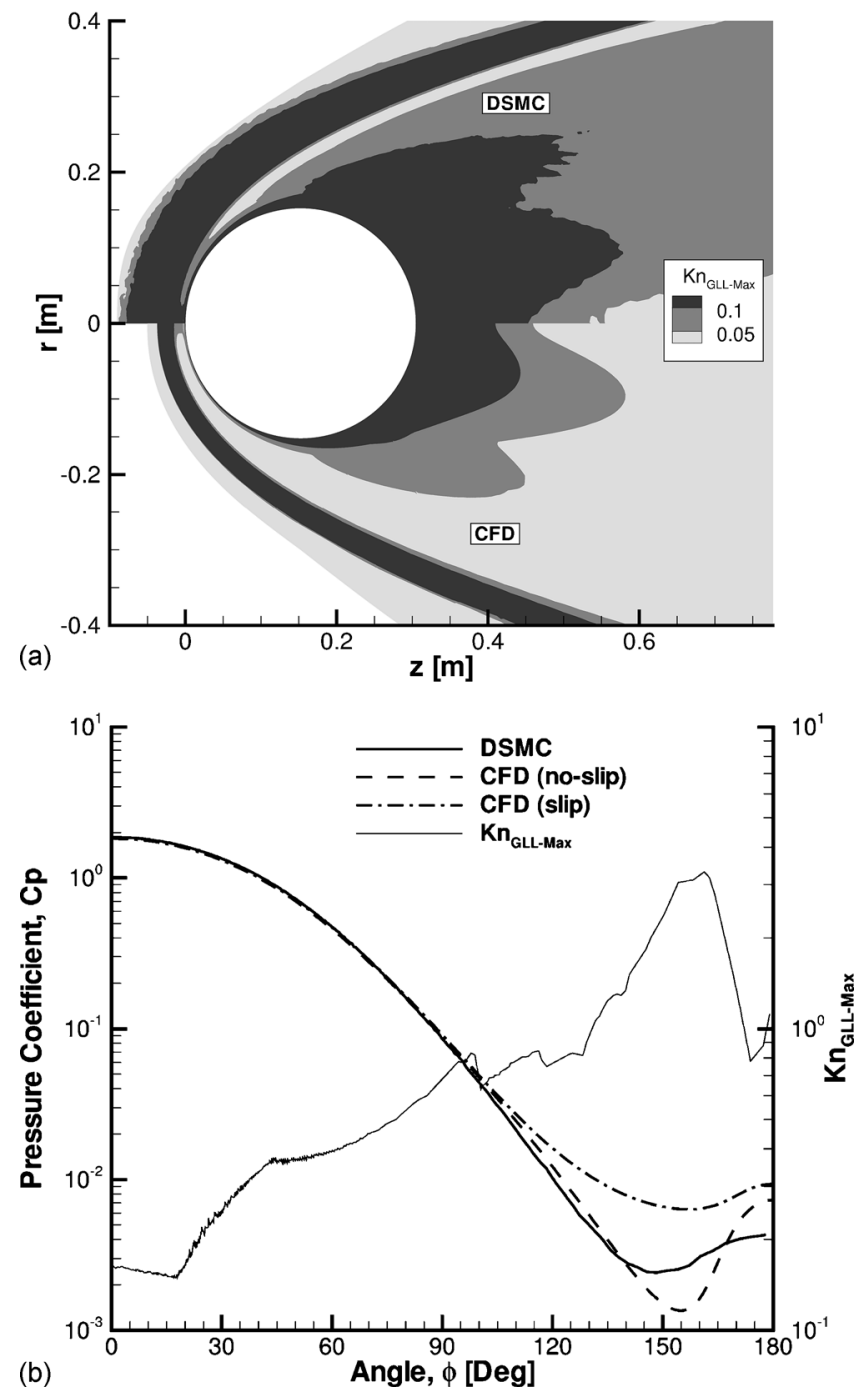

FIG. 8. $\mathrm{Kn}_{\infty}=0.01, \mathrm{Kn}_{G L L}$ contours, surface pressure (left axis), and $\mathrm{Kn}_{G L L}$ surface profile (right axis) on a sphere in a Mach 25 flow of reacting air.

Fig. 10(a). The large discrepancies between CFD and DSMC in mass fraction for nitric oxide, atomic oxygen, and atomic nitrogen that develop moving away from the stagnation point are caused by diffusion in DSMC. From this figure, it can be seen that molecular oxygen has dissociated, while molecular nitrogen has undergone little change. There is a negligible amount of atomic nitrogen and nitric oxide created behind the shock, with peak mass fractions of approximately 0.03 and 0.02 , respectively. It is an expected result that molecular oxygen dissociates more easily due to a weaker bond as compared to molecular nitrogen. The profiles for the temperatures and gradient length local Knudsen number along the stagnation streamline are given in Fig. 10(b). Since DSMC has a thicker shock, it can be seen that the temperature starts rising earlier then CFD. The flow is forced into thermal equilibrium as it moves closer to the wall, but CFD overshoots the DSMC temperatures prior to reaching equilibrium. This phenomenon has been seen in a previous study ${ }^{7}$ and is caused by the fact that the flow is in thermal nonequilibrium following the shock. 

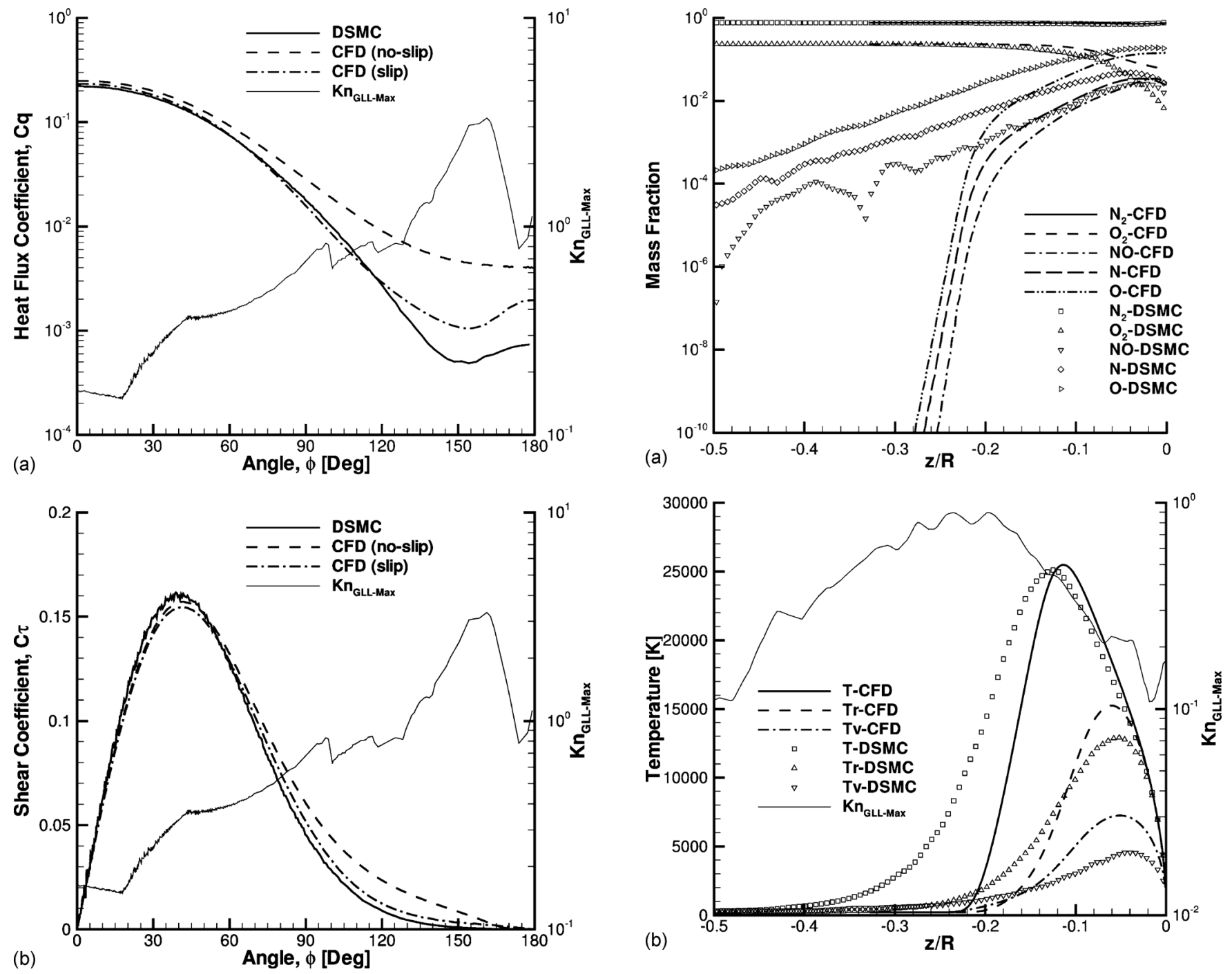

FIG. 10. $\mathrm{Kn}_{\infty}=0.01$, mass fraction (left axis), temperature (left axis), and $\mathrm{Kn}_{G L L}$ (right axis) profiles along the stagnation streamline in a Mach 25 flow of reacting air.

FIG. 9. $\mathrm{Kn}_{\infty}=0.01$, surface heat flux (left axis), surface shear stress (left axis), and $\mathrm{Kn}_{G L L}$ surface profile (right axis) on a sphere in a Mach 25 flow of reacting air.

\section{C. $\mathrm{Kn}_{\infty}=0.05$}

At a global Knudsen number of 0.05 , the flow is expected to be outside the continuum regime and in the transition regime. The contours of gradient length local Knudsen number are given in Fig. 11(a). It can be seen that the gradient length local Knudsen number is over the 0.05 limit, indicating continuum breakdown in the shock and wake.

It can be seen that the CFD method, with or without slip boundary conditions, compares well with the DSMC surface pressure coefficient over the fore body, as shown in Fig. 11(b). It is interesting to note the CFD with no-slip boundary conditions underpredicts DSMC over the aft body, while CFD with slip overpredicts DSMC. This figure also gives the gradient length local Knudsen number, which shows that at the surface, the value of $\mathrm{Kn}_{G L L}$ is over 0.05 , indicating the flow is in continuum breakdown. The CFD method overpredicts the heat flux over the entire surface as compared to DSMC, as shown in Fig. 12(a). There is an improvement in the agreement with DSMC when the slip boundary condition is utilized in the CFD method, but there is still a large dis-

agreement over the aft of the sphere. The shear stress coefficient, given in Fig. 12(b), is overpredicted by CFD assuming no-slip boundaries as compared to DSMC. However, when a slip boundary condition is employed in the CFD technique, there is better agreement with DSMC for the shear stress coefficient.

There is very little chemistry at this more rarefied condition with peak mass fractions of trace species less than 0.02 . The profiles for the temperatures and gradient length local Knudsen number along the stagnation streamline are given in Fig. 13. Since DSMC has a thicker shock, it can be seen that the temperature starts rising earlier then CFD. The flow is forced toward thermal equilibrium as it moves closer to the wall. However, at this higher Knudsen number, thermal equilibrium is not reached prior to the wall.

\section{D. $K \mathrm{n}_{\infty}=0.25$}

The highest global Knudsen number considered in this study is 0.25 . At this Knudsen number, the flow is in the rarefied regime, outside the range of where the CFD method 

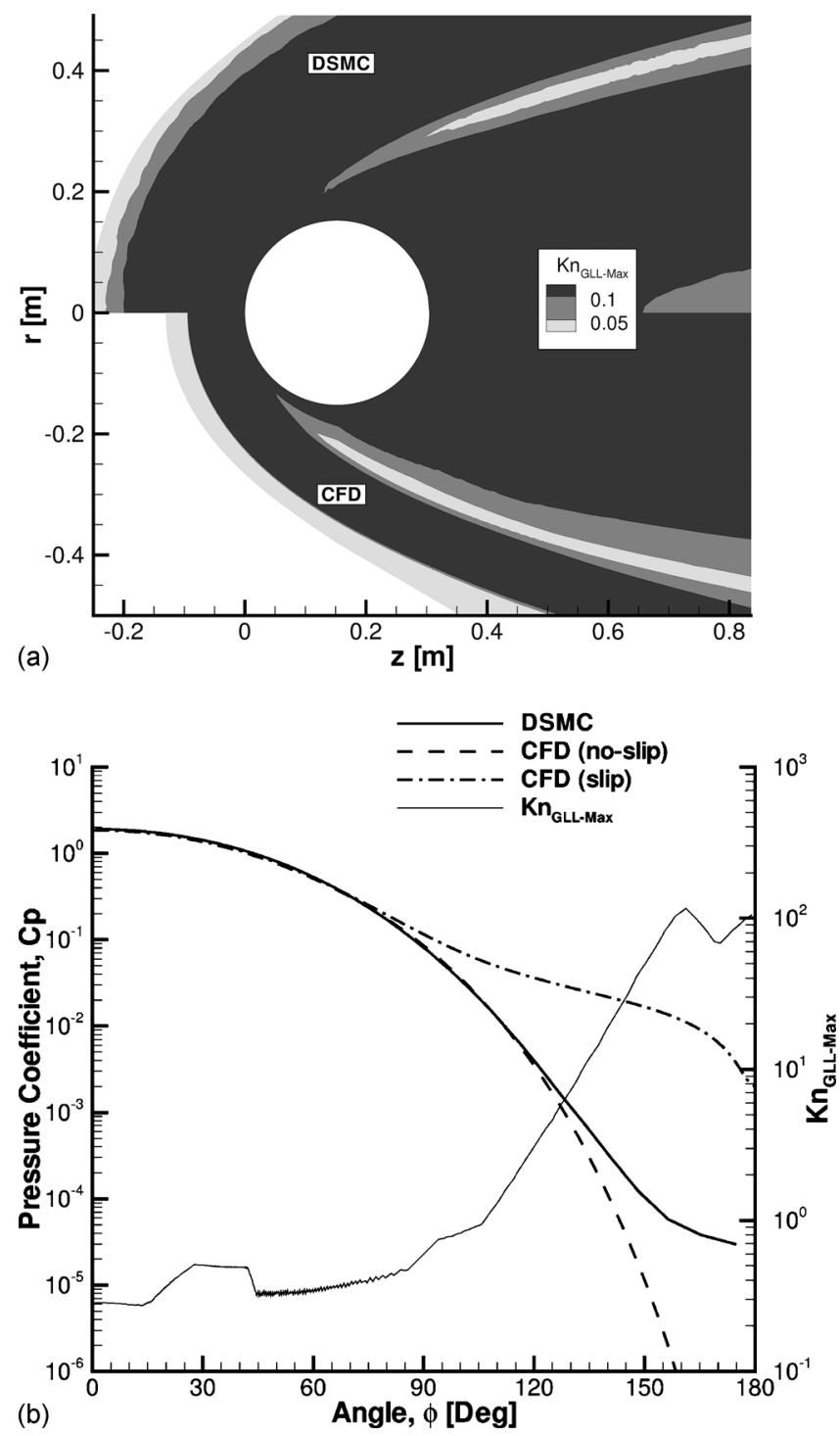

FIG. 11. $\mathrm{Kn}_{\infty}=0.05, \mathrm{Kn}_{G L L}$ contours, surface pressure (left axis), and $\mathrm{Kn}_{G L L}$ surface profile (right axis) on a sphere in a Mach 25 flow of reacting air.

should be employed. At this high of a global Knudsen number, the entire flow is in continuum breakdown, as seen in Fig. 14(a). The shock standoff distance in DSMC is farther out than in CFD, almost twice the distance in DSMC as compared to CFD.

At this condition, continuum breakdown has a strong effect on the surface properties. The surface pressure coefficient is given in Fig. 14(b), along with the surface profile of gradient length local Knudsen number. It is seen that the surface pressure coefficient is overpredicted by CFD with slip boundary conditions as compared to DSMC, while CFD with no slip underpredicts the pressure near the rear stagnation point. The surface heat flux coefficient shows very poor agreement between CFD and DSMC, as shown in Fig. 15(a). When slip boundary conditions are employed in the CFD method, there is an improvement in the heat flux results as compared to DSMC, but is overpredicted by CFD over the aft of the sphere. The shear stress is overpredicted by CFD without slip as compared to DSMC, as shown in Fig. 15(b). When slip boundary conditions are implemented in the CFD

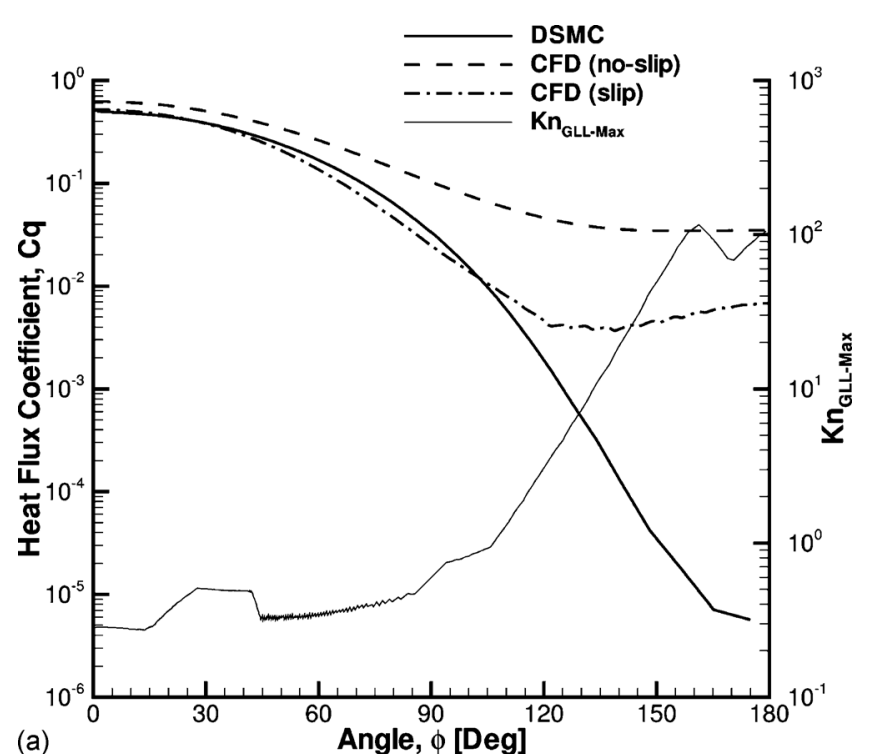

(a)

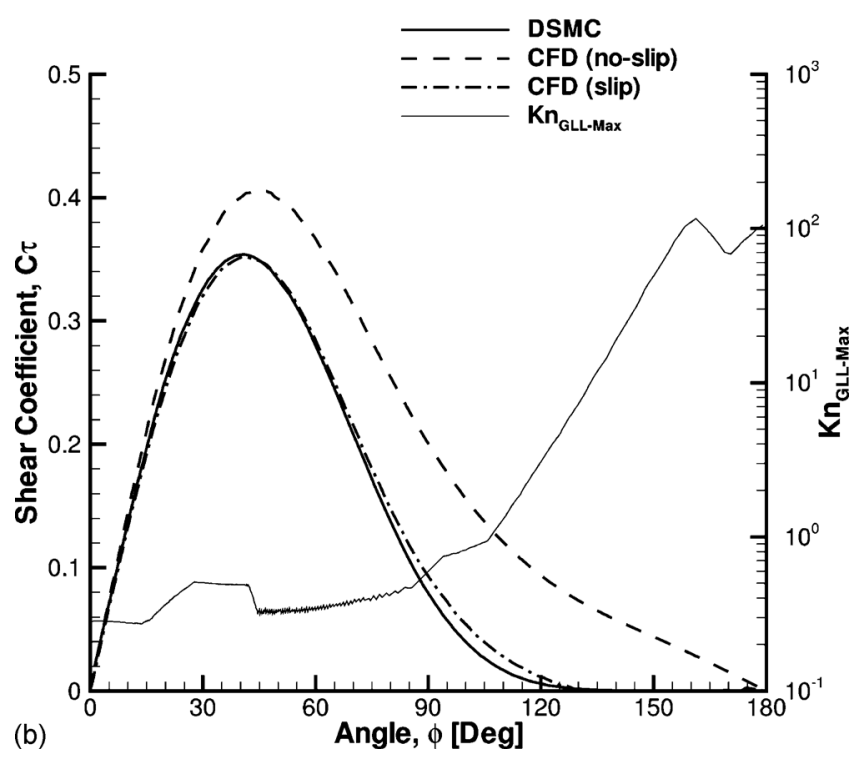

FIG. 12. $\mathrm{Kn}_{\infty}=0.05$, surface heat flux (left axis), surface shear stress (left axis), and $\mathrm{Kn}_{G L L}$ surface profile (right axis) on a sphere in a Mach 25 flow of reacting air.

method, the shear stress is underpredicted over the fore body and overpredicted over the aft body as compared to DSMC. Notice that the location of peak shear stress predicted by CFD with slip is approximately in the same location as DSMC, while the location predicted by CFD without slip occurs further back on the surface of the sphere.

The temperature and gradient length local Knudsen number profiles along the stagnation streamline are given in Fig. 16. It is interesting to note that at this global Knudsen number, the rotational and vibrational temperatures are small in comparison with the translational temperature. Since there are very few collisions, the gas never achieves thermal equilibrium. This also has the effect of limiting the amount of chemical reactions that take place in the flow, which is the reason there is almost zero dissociation in this case. 


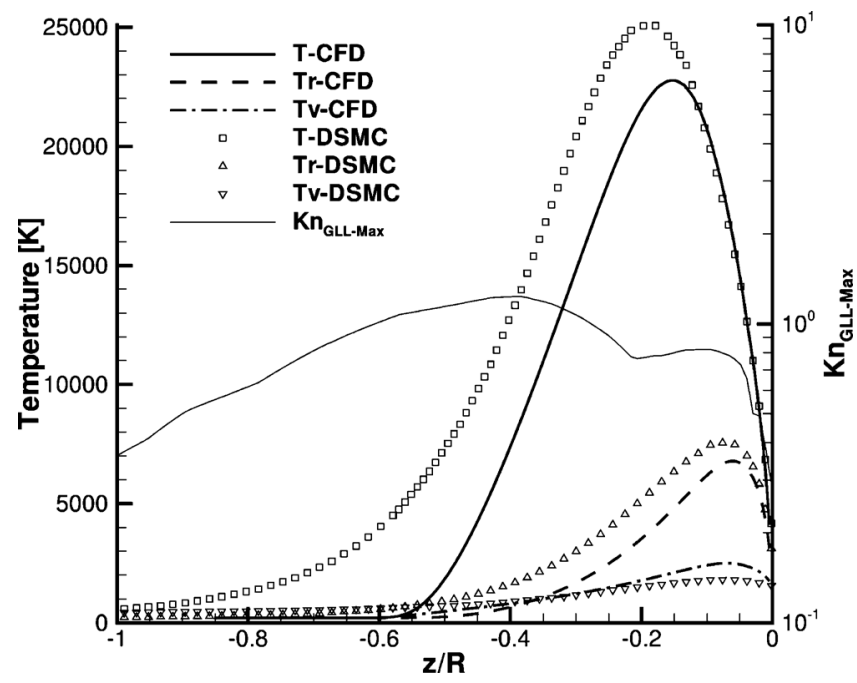

FIG. 13. $\mathrm{Kn}_{\infty}=0.05$, temperature (left axis), and $\mathrm{Kn}_{G L L}$ (right axis) profiles along the stagnation streamline in a Mach 25 flow of reacting air

\section{OVERALL TRENDS}

This section examines the stagnation pressure coefficient, the stagnation heat flux coefficient, and the drag coefficient for all cases presented in this study. This comparison is performed to be able to more easily discern the fundamental trends seen in CFD and DSMC in the cases presented in this work.

\section{A. Drag coefficient}

The integrated drag can be utilized to find the coefficient of drag, as given in Eq. (22)

$$
C_{d}=\frac{D}{\frac{1}{2} \rho_{\infty} U_{\infty}^{2} A},
$$

where $D$ is the integrated drag, $\rho$ is the density, $U$ is the velocity, $\infty$ indicates free stream conditions, and $A$ is the surface area. The coefficient of drag for each case as predicted by the CFD method, the CFD procedure with slip boundary conditions and the DSMC technique are given in Fig. 17.

The upper limit is found to be 2 using free molecular theory $^{31}$ and the lower limit is found to be 0.95 from experimental results. ${ }^{32}$ From this figure, it can be seen that all cases are in good agreement at the lower Knudsen numbers. At Knudsen numbers higher than 0.05 , the CFD method predicts the drag coefficient to be higher than the upper limit, verifying that the CFD method is only accurate in near continuum flow. It can also be observed that the CFD method with slip boundary conditions improves the agreement with DSMC. The DSMC predicted values of drag coefficient are bound by the limits and approach the upper limit as the Knudsen number increases.

\section{B. Stagnation pressure coefficient}

It was observed that the surface pressure was least affected by continuum breakdown, as it usually agreed well

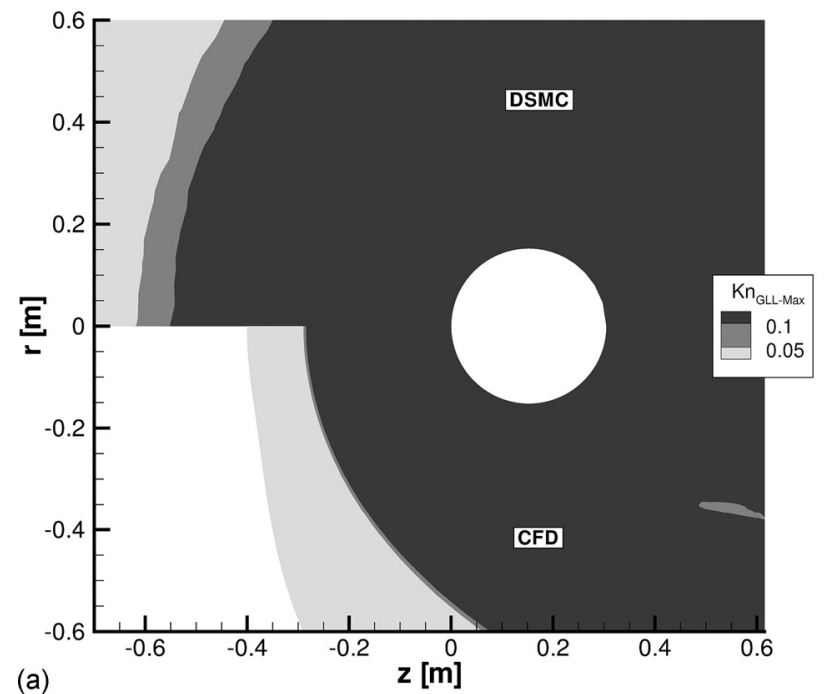

(a)

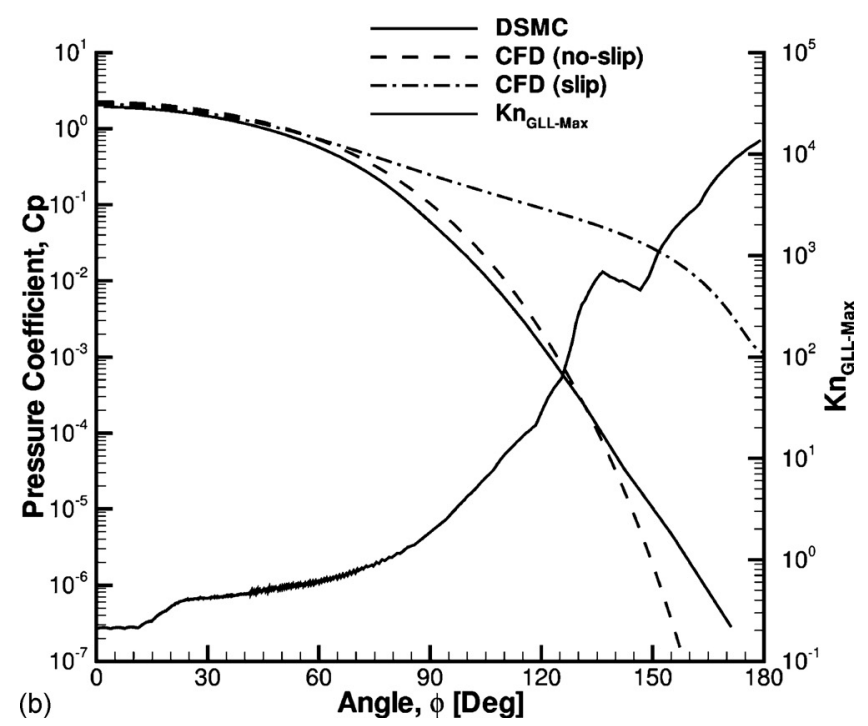

FIG. 14. $\mathrm{Kn}_{\infty}=0.25, \mathrm{Kn}_{G L L}$ contours, surface pressure (left axis), and $\mathrm{Kn}_{G L L}$ surface profile (right axis) on a sphere in a Mach 25 flow of reacting air.

between the two numerical methods. However, the surface pressure at the stagnation point did vary with the Knudsen number as shown in Fig. 18.

In this figure, the stagnation pressure coefficient from DSMC and CFD, with and without slip boundary conditions, are compared. The upper limit is found to be 2.01 using free molecular theory and the lower limit is found to be 1.825 in the inviscid limit. At lower Knudsen numbers, all the methods are in good agreement. However, as the Knudsen number increases, the agreement between the CFD method and the DSMC method diverges. At Knudsen numbers larger than 0.05 , the CFD method predicts a stagnation pressure coefficient larger than the upper limit. From the figure, it can be observed that the CFD method utilizing slip boundary conditions improves agreement with the DSMC prediction of the stagnation pressure coefficient. It is interesting to note that when the CFD method is past breakdown, it tends to overpredict DSMC, while the CFD method with slip boundary conditions is unpredictable. Enforcing the no-slip boundary condition will always give higher gradients at the wall and 

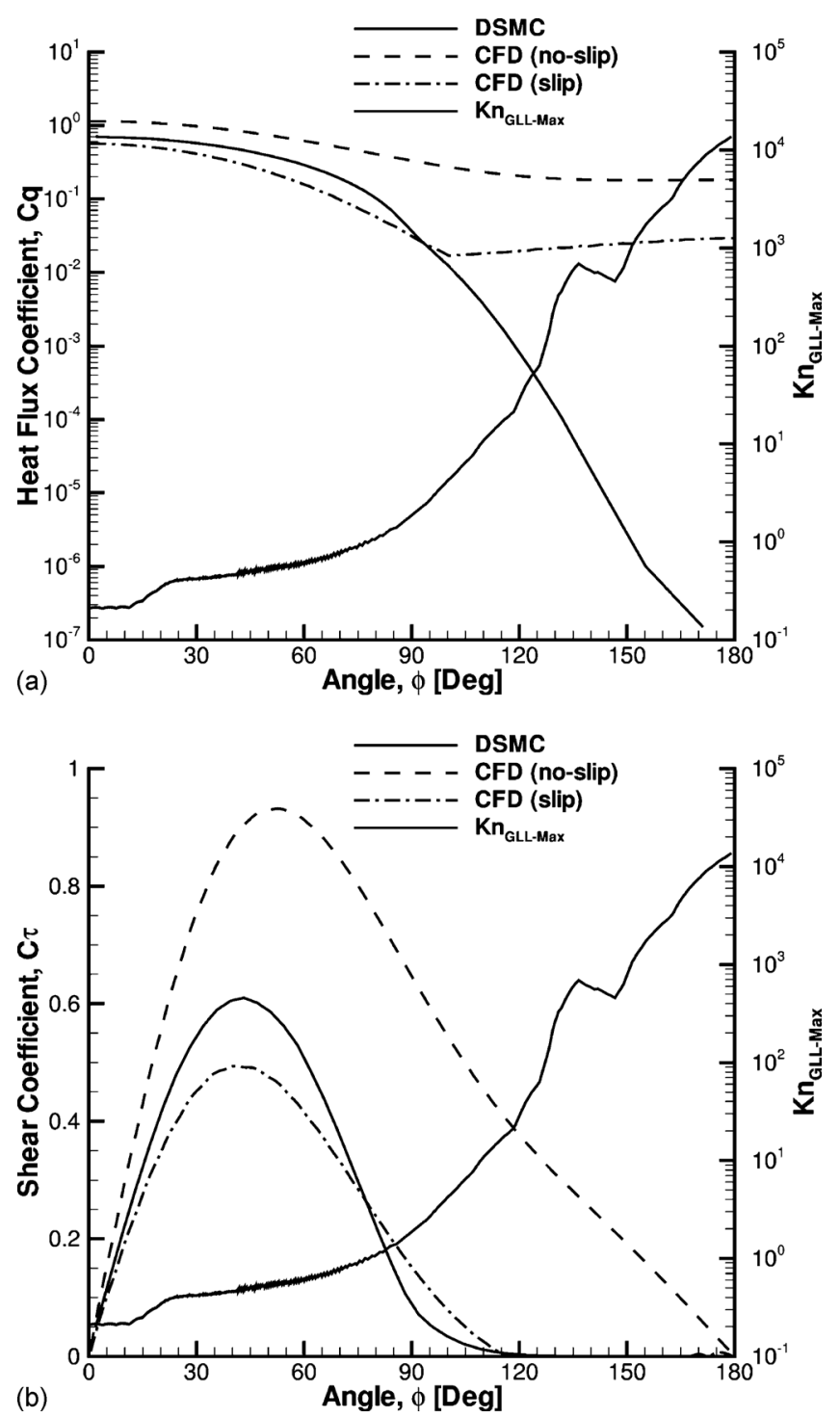

FIG. 15. $\mathrm{Kn}_{\infty}=0.25$, surface heat flux (left axis), surface shear stress (left axis), and $\mathrm{Kn}_{G L L}$ surface profile (right axis) on a sphere in a Mach 25 flow of reacting air.

hence CFD will always overpredict DSMC when in continuum breakdown. However, when the slip boundary condition is utilized, the values of velocity and temperature jump at the wall are now nonzero, causing the gradient at the wall to decrease, giving a better comparison to DSMC. It can be observed from this figure that the DSMC prediction of the stagnation coefficient of pressure is bounded by the limits and is approaching the upper limit with increasing Knudsen number.

\section{Stagnation Heat Rate Coefficient}

The maximum heat flux occurs at or near the stagnation point. The stagnation coefficient of heat flux for each case as predicted by the CFD method, the CFD procedure with slip boundary conditions, and the DSMC technique are given in Fig. 19.

In this figure, the stagnation heat flux coefficient from DSMC and CFD, with and without slip boundary conditions,

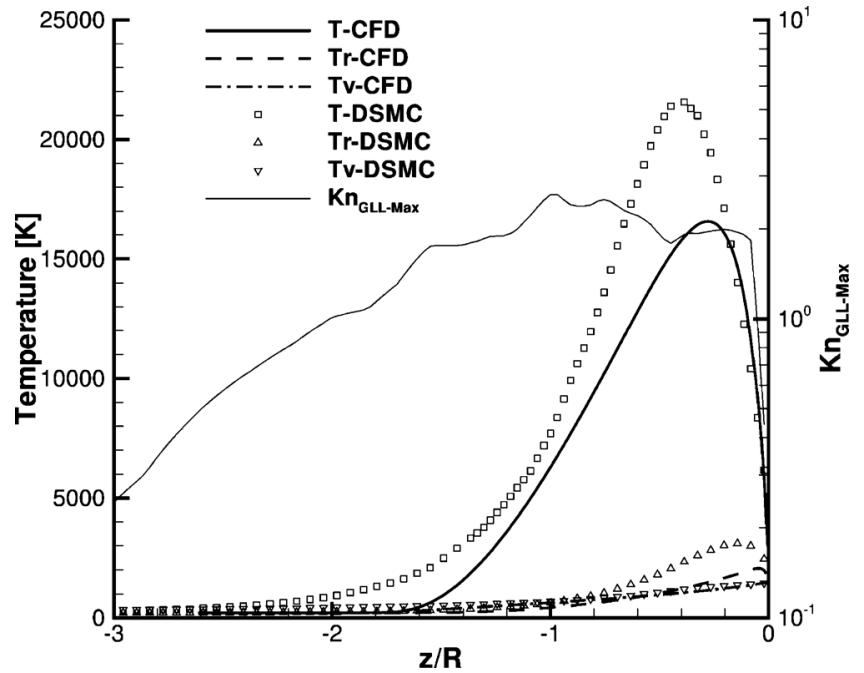

FIG. 16. $\mathrm{Kn}_{\infty}=0.25$, temperature (left axis), and $\mathrm{Kn}_{G L L}$ (right axis) profiles along the stagnation streamline in a Mach 25 flow of reacting air.

are compared. The upper limit is found to be 1 using free molecular theory and the lower limit is found to be 0.09 using a Fay-Riddell analysis. ${ }^{33}$ At lower Knudsen numbers, all the methods are in good agreement and are very close to the lower limit. As the Knudsen number increases, the disparity between the values predicted by the CFD and DSMC methods widen. At Knudsen numbers larger than 0.05, the CFD method predicts stagnation heat flux coefficients to be larger than the upper limit. Implementation of slip boundary conditions in the CFD method improves the agreement with DSMC in all cases. From the figure, it can be observed that the DSMC method stays bounded within the limits and approaches the upper limit with increasing Knudsen number.

While slip boundary conditions can allow CFD to be run at higher Knudsen numbers, it will still succumb to continuum breakdown because it only attempts to fix this problem at the surface. Continuum breakdown happens in the flow field, most notably in the shock and wake of a hypersonic object. The shear stress and heat flux are assumed to be linear functions of macroscopic flow gradients. In noncon-

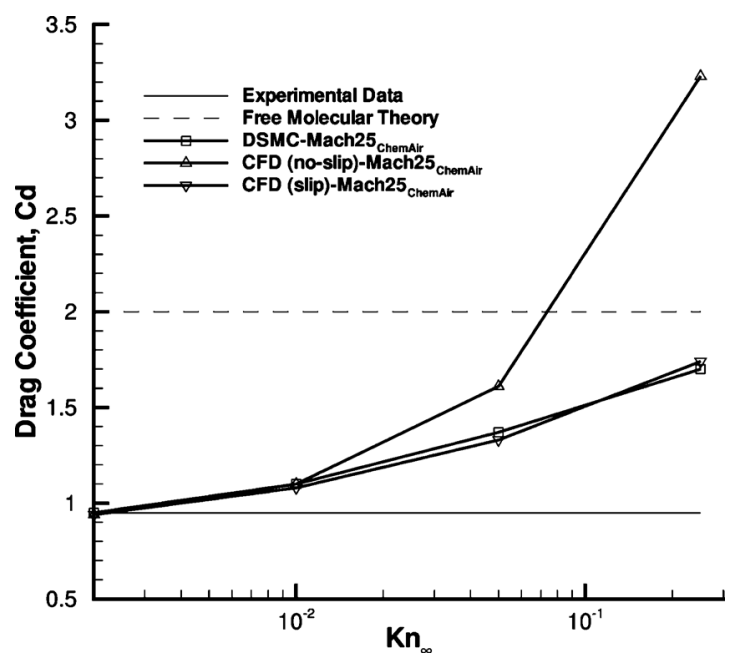

FIG. 17. Drag coefficient for DSMC and CFD with upper and lower bounds. 


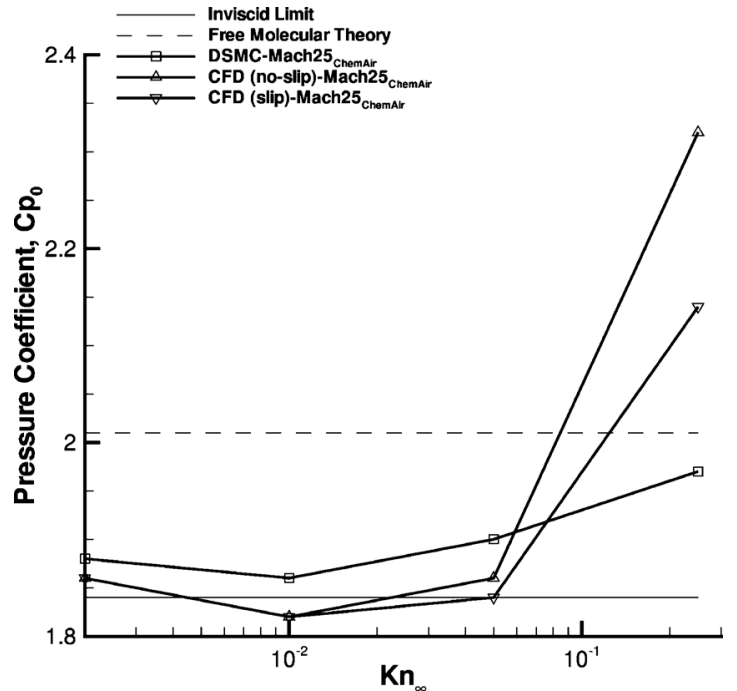

FIG. 18. Stagnation pressure coefficient for DSMC and CFD with free molecular and continuum limits.

tinuum flow, these gradients can occur over a few mean free paths, making the linear assumption invalid. Mass, momentum, and energy are physically carried by particles and transferred from particle to particle through collisions, which may be a completely nonlinear process. This means that at higher Knudsen numbers, CFD with or without slip boundary conditions will not be able to accurately predict the environment around the vehicle, including the surface properties. This is clearly seen in the stagnation pressure coefficient where there is good agreement between CFD and DSMC up until a Knudsen number of 0.25 .

\section{CONCLUSIONS}

This study investigated the effects of continuum breakdown on the surface aerothermodynamic properties (pressure, stress, and heat transfer rate) in Mach 25 flow of reacting air over a $12 \mathrm{in.} \mathrm{diameter} \mathrm{sphere} \mathrm{in} \mathrm{flow} \mathrm{regimes} \mathrm{varying}$ from continuum to rarefied. This investigation added the complexity of thermochemical nonequilibrium to earlier studies. However, before these simulations were run, the chemistry models in both numerical techniques were investigated to ensure they agree as well as possible. A threetemperature model was introduced into the CFD method for the chemical rate calculations to be able to include the rotational temperature in the rate calculations. The preferential dissociation model was modified to not only include rotational as well as vibrational energy, but also data extracted from the DSMC method. Finally, a chemical equilibrium model was included in the DSMC method to ensure that the reverse chemical rates are calculated in the same manner as the CFD method. Differences in peak heat flux and integrated drag between CFD and DSMC were observed to grow with growing global Knudsen number. When slip boundary conditions were employed in the CFD method, the agreement with DSMC improved. It was found that at higher Knudsen numbers, the effects of chemically reacting flow on continuum breakdown and the surface properties of a hypersonic body were almost nonexistent. This is due to the dif-

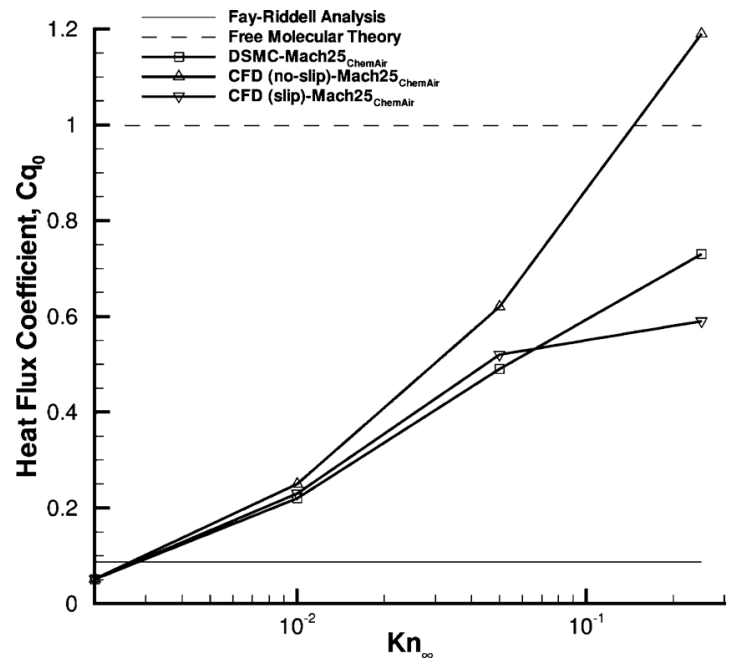

FIG. 19. Stagnation heat flux coefficient for DSMC and CFD with free molecular and continuum limits.

fuse nature of the gas at high Knudsen numbers, where collisions are infrequent and reactions are even more rare. It was found that at lower Knudsen numbers, the flow has a significant amount of chemistry occurring in the flow field.

This study summarized the trends seen in the results by examining the stagnation pressure coefficient, the stagnation heat flux coefficient, and the drag coefficient for all cases presented in this work. These comparisons are performed to be able to easily discern the fundamental differences seen in CFD and DSMC simulations presented in this study. It was seen in all three properties that the CFD method overpredicted the upper limit as the Knudsen number increased. The reason for this is due to the fact that heat flux and shear stress are directly dependent on gradients at the wall, while pressure is calculated from primitive variables, temperature and density, that do not require additional gradients. These gradients are always overpredicted in CFD, with no-slip boundary conditions, as compared to DSMC. Slip boundary conditions allow velocity and temperature jump at the surface to be nonzero, decreasing the gradients at the wall lowering the integrated drag and peak heating giving better comparisons to DSMC. The predictions by the DSMC method are always bounded by the upper and lower limits.

\section{ACKNOWLEDGMENTS}

This work is sponsored under a NASA graduate student researchers program fellowship (Grant No. NNX07AV91H) through NASA Ames Research Center monitored by Dr. Michael Wright. The use of supercomputing resources from the University of Michigan and NASA is essential to this investigation and is greatly appreciated.

${ }^{1}$ W. G. Vincenti and C. H. Kruger, Introduction to Physical Gas Dynamics (Krieger, Malabar, FL, 1965).

${ }^{2}$ G. A. Bird, Molecular Gas Dynamics and the Direct Simulation of Gas Flows (Oxford University Press, Oxford, 1994).

${ }^{3}$ I. D. Boyd, G. Chen, and G. V. Candler, "Predicting failure of the continuum fluid equations in transitional hypersonic flows," Phys. Fluids 7, 210 (1995). 
${ }^{4}$ A. J. Lofthouse, L. C. Scalabrin, and I. D. Boyd, "Velocity slip and temperature jump in hypersonic aerothermodynamics," J. Thermophys. Heat Transfer 22, 38 (2008).

${ }^{5}$ A. J. Lofthouse, I. D. Boyd, and M. J. Wright, "Effects of continuum breakdown on hypersonic aerothermodynamics," Phys. Fluids 19, 027105 (2007).

${ }^{6}$ A. J. Lofthouse, L. C. Scalabrin, and I. D. Boyd, "Hypersonic aerothermodynamics analysis across nonequilibrium regimes using continuum and particle methods," AIAA Paper No. 2007-3903, 2007.

${ }^{7}$ T. D. Holman and I. D. Boyd, "Effects of continuum breakdown on the surface properties of hypersonic sphere," J. Thermophys. Heat Transfer 23, 660 (2009).

${ }^{8}$ S. Dietrich and I. D. Boyd, "Scalar and parallel optimized implementation of the direct simulation Monte Carlo method," J. Comput. Phys. 126, 328 (1996).

${ }^{9}$ K. C. Kannenberg and I. D. Boyd, "Strategies for efficient particle resolution in the direct simulation Monte Carlo method," J. Comput. Phys. 157, 727 (2000).

${ }^{10}$ L. C. Scalabrin and I. D. Boyd, "Development of an unstructured NavierStokes solver for hypersonic nonequilibrium aerothermodynamics," AIAA Paper No. 2005-5203, 2005.

${ }^{11}$ L. C. Scalabrin and I. D. Boyd, "Numerical simulation of weakly ionized hypersonic flow for reentry configurations," AIAA Paper No. 2006-3773, 2006.

${ }^{12}$ C. R. Wilke, "A viscosity equation for gas mixtures," J. Chem. Phys. 18, 517 (1950)

${ }^{13}$ R. N. Gupta, J. M. Yos, R. A. Thompson, and K. Lee, "A review of reaction rates and thermodynamic and transport properties for an 11species air model for chemical and thermal nonequilibrium calculations to 30,000 K," NASA Technical Report No. 1232, 1990.

${ }^{14}$ G. E. Palmer and M. J. Wright, "Comparison of methods to compute high-temperature gas thermal conductivity," AIAA Paper No. 2003-3913, 2003.

${ }^{15}$ G. E. Palmer and M. J. Wright, "Comparison of methods to compute high-temperature gas viscosity," J. Thermophys. Heat Transfer 17, 232 (2003).

${ }^{16}$ M. J. Wright, D. Bose, G. E. Palmer, and E. Levin, "Recommended collision integrals for transport property computations, Part 1: Air Species," AIAA J. 43, 2558 (2005).
${ }^{17}$ I. D. Boyd, "Analysis of vibrational-translational energy transfer using the direct simulation Monte Carlo method," Phys. Fluids A 3, 1785 (1991).

${ }^{18}$ R. C. Millikan and D. R. White, "Systematics of vibrational relaxation," J. Chem. Phys. 39, 3209 (1963).

${ }^{19}$ C. Park, Nonequilibrium Hypersonic Aerothermodynamics (Wiley, New York, 1990).

${ }^{20}$ N. E. Gimelshein, S. F. Gimelshein, and D. A. Levin, "Vibrational relaxation rates in the direct simulation Monte Carlo method," Phys. Fluids 14, 4452 (2002).

${ }^{21}$ T. R. Deschenes, T. D. Holman, I. D. Boyd, and T. E. Schwartzentruber, "Analysis of internal energy transfer within a modular particle-continuum method," AIAA Paper No. 2009-1216, 2009.

${ }^{22}$ I. D. Boyd, "Modeling backward chemical rate processes in the direct simulation Monte Carlo method," Phys. Fluids 19, 126103 (2007).

${ }^{23}$ B. L. Haas and I. D. Boyd, "Models for direct Monte Carlo simulation of coupled vibration-dissociation," Phys. Fluids A 5, 478 (1993).

${ }^{24}$ I. D. Boyd and T. Gokcen, "Computation of axisymmetric and ionized flows using particle and continuum methods," AIAA J. 32, 1828 (1994).

${ }^{25}$ C. Park, "The limits of two-temperature model," AIAA Paper No. 2010911, 2010.

${ }^{26}$ C. Park, "A review of reaction rates in high temperature air," AIAA Paper No. 89-1740, 1989.

${ }^{27}$ C. Park, "Review of chemical-kinetic problems of future NASA missions, I: Earth entries," J. Thermophys. Heat Transfer 7, 385 (1993).

${ }^{28}$ C. Park, "Review of chemical-kinetic problems of future NASA missions, II: Mars entries," J. Thermophys. Heat Transfer 8, 9 (1994).

${ }^{29}$ B. W. Silverman, Density Estimation for Statistics and Data Analysis (Chapman and Hall, London, 1986).

${ }^{30}$ F. E. Lumpkin, B. L. Haas, and I. D. Boyd, "Resolutions of differences between collision number definitions in particle and continuum simulations," Phys. Fluids A 3, 2282 (1991).

${ }^{31}$ T. I. Gombosi, Gaskinetic Theory (Cambridge University Press, Cambridge, 1994).

${ }^{32}$ A. B. Bailey and J. Hiatt, "Free-flight measurements of sphere drag at subsonic, transonic, supersonic, and hypersonic speeds for continuum, transition, and near-free-molecular flow conditions," AEDC Technical Report No. 291, 1970.

${ }^{33}$ J. J. Bertin, Hypersonic Aerothermodynamics (American Institute of Aeronautics and Astronautics, Washington, DC, 1994). 